\title{
Day-ahead and Intraday Dispatch of an Integrated Biomass-Concentrated Solar System: A Multi-Objective Risk-Controlling Approach
}

\author{
Hooman Khaloie, François Vallée, Member, IEEE, Chun Sing Lai, Senior Member, IEEE, Jean-François Toubeau, \\ Member, IEEE, and Nikos D. Hatziargyriou, Fellow, IEEE
}

\begin{abstract}
Solar energy and bioenergy are two leading renewable forms of energy in the move toward a near-zeroemission electric power industry. Concentrated solar power units coupled with thermal storage and biomass power plant offer dispatchable electricity, raising their ever-growing role in future renewable-dominated networks. This paper proposes a dayahead and intraday dispatch model for maximizing the profit of an Integrated Biomass-Concentrated Solar (IBCS) system considering the synergies arising from their coupled operation. To sensibly capture uncertainty and decision sequence of real-life electricity markets, a two-stage stochastic structure is proposed, while the solar-related uncertainty is involved using Information Gap Decision Theory (IGDT). The model is complemented with a novel multi-objective architecture based on the compound of IGDT and Conditional Value-at-Risk (CVaR), which allows handling risk exposure to both stochastic and IGDT inputs. The Pareto strategies in the multi-objective model are extracted through an expanded form of the $\epsilon$-constraint method, whereas a posteriori approach based upon the out-of-sample assessment is applied to derive the optimal dispatch pattern among the generated Pareto strategies. The simulation results demonstrate that: 1) the proposed integrated dispatch model achieves substantial profitability, and 2) the performance of the suggested CVaRIGDT model is superior to conventional approaches.
\end{abstract}

Index Terms-Day-ahead and intraday dispatch, Integrated Biomass-Concentrated Solar (IBCS) system, out-of-sample assessment, risk management.

\section{NOMENCLATURE}

A. Sets and Indices

$\mathcal{R}($.

Uncertainty set of thermal power in solar field using the IGDT-based technique.

$s(\mathcal{S}), t(\mathcal{T}) \quad$ Indices (Sets) of scenario and time.

\section{B. Parameters}

$C^{\mathrm{b}}$

$C a^{\mathrm{b}}$

Cost of biomass feedstock [€/ton].

$\bar{G}_{t}^{\mathrm{f}}$

$\overline{G G}$ Calorific value of biomass feedstock [MWh/ton].

Forecasted thermal power of the solar field [MW].

Thermal power capacity of the solar field [MW].

H. Khaloie, F. Vallée, and J.-F. Toubeau are with the Power Systems and Markets Research Group, University of Mons, Mons, Belgium.

C. S. Lai (corresponding author) is with the Brunel Interdisciplinary Power Systems Research Centre, Department of Electronic and Electrical Engineering Brunel University London, London, UB8 3PH, U.K (e-mail: chunsing.lai@brunel.ac.uk).

N. D. Hatziargyriou is with the School of Electrical and Computer Engineering, the National Technical University of Athens, Athens, Greece.
$M^{\mathrm{b}} \quad$ Maximum amount of available biomass feedstock within the scheduling horizon [ton].

$p \quad$ Number of equally distributed parts in the Pareto frontier.

STD Standard deviation.

$t^{\text {off,bo }}, t^{\text {off,pb }}$ Minimum down-time of biomass boiler and power block [hr].

$t^{\text {on,bo }}, t^{\text {on,pb }}$ Minimum up-time of biomass boiler and power block [hr].

$\beta^{\text {scheme }_{1,2}} \quad$ Expected profit deviation factor in the IGDTbased technique for risk-controlling schemes 1 and 2.

$\gamma^{\mathrm{SU}} \quad$ Required thermal power for start-up the power block [MW].

$\gamma_{0}^{\mathrm{TS}} \quad$ Available energy in the thermal storage at the

beginning of the scheduling horizon [MWh]

$\underline{\Gamma^{b o}}, \overline{\Gamma^{\text {bo }}} \quad$ Minimum and maximum thermal power lim-

$\underline{\Gamma^{\mathrm{pb}}}, \overline{\Gamma^{\mathrm{pb}}}$ its of the biomass boiler [MW].

$\underline{\Gamma^{\mathrm{Ts}}}, \overline{\Gamma^{\mathrm{Ts}}}$

$\Delta^{\text {down }}, \Delta^{\text {up }}$ Minimum and maximum thermal power limits of the power block [MW].

Minimum and maximum limits of the energy stored in thermal storage [MWh].

Ramp-down and ramp-up limits of the power block [MW/hr].

$\overline{\zeta^{\mathrm{ch}}}, \overline{\zeta^{\mathrm{dis}}}$

$\eta^{\mathrm{bo}}, \eta^{\mathrm{pb}}$

$\eta^{\mathrm{ch}}, \eta^{\mathrm{dis}}$

$\kappa$

$\varkappa_{t, s}$

$\Lambda$

$\overline{\Xi \mathrm{pb}}$

$\rho$

$\sigma_{t, s}^{\mathrm{DA}}, \sigma_{t, s}^{\mathrm{I}}$ $\Upsilon$

Maximum charging and discharging rates [MW].

Efficiency of biomass boiler and power block. Efficiency of thermal storage during charging and discharging.

Confidence level in CVaR theory.

Parameter indicating the difference between intraday and day-ahead prices.

Risk-averse parameter in conventional CVaR model.

Electric power capacity of the power block [MW].

Hourly thermal energy loss in thermal storage.

Day-ahead and intraday prices [€/MWh].

Parameter limiting the intraday power dispatch.

C. Variables

$\mathrm{CVaR}_{\kappa}, \Pi \quad \mathrm{CVaR}$ in confidence level $\kappa$ and expected profit of the integrated system $[€]$. 


\begin{tabular}{|c|c|}
\hline$\frac{f}{t}$ & $\begin{array}{l}\text { Uncertain thermal power of the solar fie } \\
{[\mathrm{MW}] \text {. }}\end{array}$ \\
\hline $\mathrm{b}$ & $\begin{array}{l}\text { Mass of biomass feedstock injected to t } \\
\text { biomass boiler [ton]. }\end{array}$ \\
\hline & The $\epsilon$ th range of $\alpha^{\text {scheme }_{1}}$ or $\alpha^{\text {scheme }_{2}}$. \\
\hline & $\begin{array}{l}\text { Binary variable indicating the start-up of } \\
\text { power block. }\end{array}$ \\
\hline${ }_{t}^{\mathrm{bo}}, u_{t}^{\mathrm{pb}}$ & $\begin{array}{l}\text { Commitment statuses of biomass boiler a } \\
\text { power block. }\end{array}$ \\
\hline$z_{t}^{\mathrm{ch}}, z_{t}^{\mathrm{dis}}$ & $\begin{array}{l}\text { Commitment statuses of thermal storage } \\
\text { charging and discharging. }\end{array}$ \\
\hline$t^{\text {scheme }_{1,2}}$ & $\begin{array}{l}\text { IGDT-based } \\
\text { schemes } 1 \text { anc }\end{array}$ \\
\hline bo & $\begin{array}{l}\text { Thermal power output of the biomass bo } \\
\text { [MW]. }\end{array}$ \\
\hline$\gamma_{t, s}^{\mathrm{h}, \mathrm{bo}}, \gamma_{t, s}^{\mathrm{h}, \mathrm{f}}$ & $\begin{array}{l}\text { Thermal power transferred from bioma } \\
\text { boiler and solar field to the thermal stora } \\
\text { [MW]. }\end{array}$ \\
\hline & $\begin{array}{l}{ }_{,} \text {Thermal power transferred directly fr } \\
\text { biomass boiler, solar field, and thermal } \\
\text { age to the power block [MW]. }\end{array}$ \\
\hline & Energy level of the thermal storage [MWh]. \\
\hline & Extra variable used for $\epsilon$-constraint appr \\
\hline IIts & $\begin{array}{l}\text { Day-ahead and intraday power dispatc } \\
\text { [MW]. }\end{array}$ \\
\hline$\xi_{t, s}^{\mathrm{pb}}, \gamma_{t, s}^{\mathrm{pb}}$ & $\begin{array}{l}\text { Electric and thermal power of the power } \\
\text { block [MW]. }\end{array}$ \\
\hline & $\begin{array}{l}\text { selectric power generated directly fro } \\
\text { biomass boiler, solar field, and thermal st } \\
\text { age [MW]. }\end{array}$ \\
\hline$I_{0}$ & $\begin{array}{l}\text { Expected profit of the integrated system cor- } \\
\text { responding to } \bar{G}_{t}^{\mathrm{f}} \text {. }\end{array}$ \\
\hline & Extra variables used for CVaR modeling. \\
\hline & I I \\
\hline
\end{tabular}

\section{INTRODUCTION}

$\mathbf{T}$ HE significance of renewable energy sources, such as solar and bioenergy, is constantly increasing to fulfill the ambitious goals of policymakers for greenhouse gas emission mitigation and moving toward a sustainable electricity supply [1]. The electricity produced by solar energy however is intermittent, posing important challenges to the power system operators for its accommodation [2]. Nevertheless, emerging technologies like concentrated solar power units, similar to biomass energy resources, do not suffer from this deficiency, while they are able to generate dispatchable electric power, facilitating their integration into power systems.

The literature related to the participation of biomass energy resources in the power system operation and the electricity markets is somehow limited and disparate [3]-[8]. In [3], Panos and Kannan investigated the long-term role of domestic biomass in Switzerland's electricity and heat markets. Lai and McCulloch [4] researched the optimal sizing of a biomasssolar system with energy storage. In [5], Li et al. explored the optimal scheduling of a combined heat and power-based energy system with solar and biogas energy sources in order to mitigate the dependence on batteries in remote regions. Yang et al. [6] introduced an expansion planning model for the coupled electric power system and biomass delivery networks using a multi-stage stochastic programming framework. In [7], Blanco et al. developed a self-scheduling model for combined heat and power systems in the day-ahead market considering wood chips and natural gas boilers for the intended combined heat and power system. Wang [8] et al. proposed a scheduling model for optimal operation of an integrated solarbiomass power plant. This is one of the few published works relevant to large-scale biomass energy resources participating in electricity markets.

Many studies have focused on the contribution of concentrated solar power units in power systems [9]- [13]. Du et al. [9] established a four-day unit commitment model for a power system equipped with concentrated solar power units. In [10], Wu et al. presented a profit-allocation algorithm for wind farms and concentrated solar units jointly participating in electricity markets using a stochastic model. Zhao et al. [11] provided an Information Gap Decision Theory (IGDT)-based structure to tackle the self-scheduling problem of concentrated solar power units and various responsive loads. In [11], market prices, solar- and demand response-involved uncertainties were handled by the IGDT technique. Khaloie et al. [12] addressed the behavior problem of concentrated solar units along with wind, demand response, and compressed air energy storage resources in electricity markets using a three-stage stochastic architecture. In [13], Zhao et al. presented a mixed Conditional Value-at-Risk (CVaR)-IGDT framework for day-ahead and real-time offering strategy of a concentrated solar power unit. It is important to note that the proposed CVaR-IGDT in this paper differentiates from the methodology suggested in [13], whereas the paradigm established in [13] suffers from fundamental deficiencies, as highlighted in the following.

Although previous papers have tackled several problems in the modeling and participation of biomass or concentrated solar systems in electricity markets, a number of weaknesses can be observed. First, the model for the optimal operation of large-scale biomass power plants in electricity markets [8] suffers from two issues: 1) The biomass power unit has been treated as a zero-cost energy resource, such as wind and photovoltaic systems, while the biomass feedstock cost accounts for a substantial portion of the operating cost [14]; 2) Simultaneous injection of two different biomass fuels was considered for the biomass unit's daily operation, while most large-scale power units are designed and operated with only one kind of biomass feedstock, especially in daily operation [14]. Despite manifold advantages stated in the literature for the hybridization of concentrated solar and biomass power units [15]- [18], the effect of such hybridization in power system studies and its behavior in electricity markets have not been adequately covered, so far. Besides, the profitability of such hybridization from the viewpoint of power producers taking part in electricity markets has not been addressed yet. The principal advantages of the solar-biomass hybridization compared to stand-alone concentrated solar and biomass systems are:

- Greater electric power output and higher thermal system 
stability [15].

- Lower capital costs [16].

- Improving the system's dispatchability and stable electricity generation [17].

- Decreasing annually biomass feedstock consumption, enhancing the entire system efficiency, and boosting maximum running hours [18].

- Reducing the size of biomass storage sites [16].

One of the paramount issues in power and energy studies is the risk-handling arising from uncertainties. There is a wide range of methods to handle uncertainties that possess their pros and cons. By benefiting from the advantages of stochastic and IGDT techniques together, the combination of stochastic-IGDT is among the promising methodologies [13]. Authors in [13] proposed a CVaR-IGDT approach using weighted sum method, but their suggested methodology had the following drawbacks: 1) The method could not directly handle the original $\mathrm{CVaR}$ index, while it relied on optimizing an extra variable, called mixed $\mathrm{CVaR}$, which is not a risk evaluation parameter; 2) The objective functions' scaling was not considered, lessening the applicability of the suggested methodology to build efficient Pareto solutions; 3) The built efficient Paretos were irregularly distanced solutions, hardening the decision-making process for the administrators; 4) The objective functions' range was not optimized and was not included in the developed methodology, resulting in unrealistic solutions; 5) The solar field's capacity was not incorporated into the formulation of the CVaR-IGDT method; 6) The authors did not work toward finding the optimal strategy; 7) The performance of the method should be evaluated through an out-of-sample assessment, while it was not covered in [13]. The significant difference between the obtained values for the after-the-fact analysis in [13] clearly illustrates the room for improvement of the developed approach. Thus, the third weakness appertains to the lack of a comprehensive and precise CVaR-IGDT model in the literature.

To fill the research gaps outlined above, this study is directed toward presenting a day-ahead and intraday dispatch pattern for an Integrated Biomass-Concentrated Solar (IBCS) system, while both systems are paired corresponding to the practical operating model of such systems in real-world applications. The day-ahead and intraday dispatch problem is affected by various uncertain data, including market prices and solar-related uncertainty. In this vein, uncertainty originating from market prices is handled through a two-stage stochastic model, whereas the IGDT procedure characterizes the uncertainty caused by solar energy. In order to accurately model the risk exposure to both stochastic and IGDT parameters, a comprehensive CVaR-IGDT model founded on lexicographic and multi-objective optimizations is proposed. To facilitate the decision-making process for the IBCS system in terms of finding the best operating strategy, a posteriori procedure relying on the out-of-sample assessment is accomplished. Finally, a validation process relying on an out-of-sample test is performed to analyze the efficiency of the hybrid CVaR-IGDT method compared to deterministic and stochastic approaches. The major contributions of this paper are summarized as follows:

1) Proposing a day-ahead and intraday dispatch model for an IBCS system considering the synergies associated with the hybridization of biomass and concentrated solar resources. For the first time in the literature, the profitability of an IBCS system participating in electricity markets is examined in this paper.

2) Feeding the model (contribution 1) by a new operating model for large-scale biomass power resources following their real-world operational structure.

3) Presenting a novel, comprehensive, and accurate CVaRIGDT model using lexicographic and $\epsilon$-constraint procedures along with executing a posteriori analysis to find the optimal dispatch plan. Moreover, the effectiveness of the proposed CVaR-IGDT model is validated through a comparative study with existing models [13].

4) Implementing in-sample and out-of-sample assessments to evaluate the efficiency of the proposed CVaR-IGDT method compared to conventional deterministic and full stochastic approaches.

The paper proceeds as following. Section [I] indicates the considered market framework and problem assumptions. Section III] presents the problem formulation of the IBCS system based on stochastic programming. The procedure of incorporating CVaR and IGDT into the stochastic formulation is discussed in Section IV. Section V is dedicated to the suggested CVaRIGDT model. Section VI presents the simulation results, and finally, conclusions are given in Section VII

\section{Market Framework And Model Assumptions}

In this paper, the market framework is in line with many European electricity markets, which possess various trading floors. Focusing on the Iberian electricity market [19], this work is founded on the participation of the IBCS system in the day-ahead and intraday markets as these markets concern the majority of daily transactions of renewable resources. The day-ahead and intraday markets are cleared at 10 a.m. and 11 p.m. of the day before energy delivery, respectively [19]. First, the IBCS system participates in the day-ahead market without knowing the day-ahead market-clearing prices. After revealing the day-ahead prices, the IBCS system takes part in the intraday market prior to understanding the behavior and outcomes of this market. Like extensive offering models developed for the Iberian electricity market, the IBCS system does not participate in the real-time market since it follows a dual pricing mechanism where the energy can only be sold at a price lower than the day-ahead market. To summarize, the market framework for the IBCS unit follows a two-stage sequential model [19].

The assumptions considered in this work are as follows: 1. The IBCS system is a price-taker agent taking part in dayahead and intraday markets. To avoid being a price-maker agent in the intraday trading floor, the intraday dispatch power is limited to a portion of its day-ahead power dispatch.

2. Like many biomass power plants running in Spain, forest residues are the feedstock used to generate electricity. The feedstock cost arises from the process of collecting and 


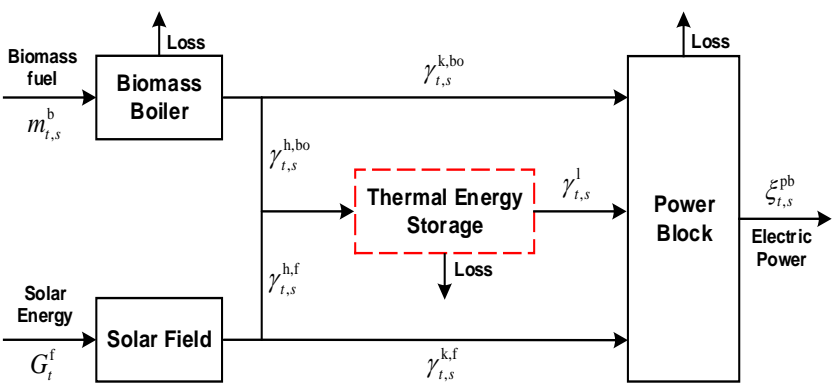

Fig. 1: Configuration of the proposed IBCS system.

transporting forest residues to the site. The feedstock cost is assumed constant. Moreover, the daily available biomass feedstock is limited [14].

3. To fully tackle the sequence of market decisions, a two-stage stochastic paradigm is adopted for uncertainty characterization of day-ahead and intraday prices. Moreover, using the CVaR index, the IBCS system can exceedingly take risk-averse actions against uncertainties originating from the markets. Given that the market prices depend on various factors such as market participants' offering and bidding strategies, equipment contingencies, renewable power generation of market players, etc., taking risk-averse actions against market uncertainties is a more established attitude compared to the risk-seeker one [19]. Therefore, in this paper, only risk-averse actions are taken when facing market uncertainty.

4. To cope with the solar-related uncertainty, the IGDT procedure is applied. The IGDT technique's superiority lies in the uncertainty handling of any parameter without perceiving its historical behavior, meaning that the IGDT procedure can be applied for any parameter for which there is a lack of sufficient historical data. Therefore, this method would be beneficial in circumstances wherein the decision-maker has less or insufficient historical data of the uncertain parameter. It is worth highlighting that enough historical data is required to model the stochastic nature of an uncertain parameter. However, there are other methods than IGDT that can be applied when there is insufficient historical data, such as fuzzy, robust, etc., whereas, among all other methods, the IGDT can take both risk-averse and risk-seeker actions against the uncertain parameter individually. In this regard, the IGDT procedure gives the IBCS system the possibility to take both risk-averse and risk-seeker actions for the uncertainty related to solar energy. Since this uncertain source merely depends on solar energy forecasting, taking both risk-averse and riskseeker actions is a well-founded practice [13], [20].

\section{Problem Formulation OF The IBCS System}

Fig. 1 presents the configuration of the IBCS system. The IBCS system comprises four main blocks: biomass boiler, solar field, thermal storage, and power block. The thermal energy produced by the biomass boiler and solar field can be transferred to the power block or stored in the thermal storage for later use. The transferred thermal energy to the power block spins a steam turbine coupled with an electric generator to produce electricity.
TABLE I: Classification of Decision Variables in the Proposed Two-Stage Stochastic Programming.

\begin{tabular}{|l|c|c|}
\hline \multicolumn{2}{|c|}{ First-stage } & Second-stage \\
\cline { 1 - 2 } Here-and-Now & Special Here-and-Now & Wait-and-See \\
\hline & & $\xi_{t, s}^{\mathrm{I}}, m_{t, s}^{\mathrm{b}}$ \\
$r_{t}, u_{t}^{\mathrm{bo}}, u_{t}^{\mathrm{pb}}$ & & $\gamma_{t, s}^{\mathrm{bo}}, \gamma_{t, s}^{\mathrm{h}, \mathrm{bo}}, \gamma_{t, s}^{\mathrm{h}, \mathrm{f}}$ \\
$z_{t}^{\mathrm{ch}}, z_{t}^{\mathrm{dis}}$ & $\xi_{t, s}^{\mathrm{DA}}$ & $\gamma_{t, s}^{\mathrm{k}, \mathrm{bo}}, \gamma_{t, s}^{\mathrm{k}, \mathrm{f}}, \gamma_{t, s}^{\mathrm{l}}$ \\
& & $\gamma_{t, s}^{\mathrm{Ts}}, \xi_{t, s}^{\mathrm{pb}}, \gamma_{t, s}^{\mathrm{pb}}$ \\
& & $\xi_{t, s}^{\mathrm{k}, \mathrm{bo}}, \xi_{t, s}^{\mathrm{k}, \mathrm{f}}, \xi_{t, s}^{\mathrm{l}}$ \\
\hline
\end{tabular}

The formulation of the day-ahead and intraday dispatch for the IBCS system using two-stage stochastic programming is addressed in the following. The first- and second-stage decisions of the IBCS system are listed in Table I Note that all binary variables fall into the here-and-now decisions, while the power offered to the day-ahead trading floor is a special hereand-now decision [21], [22]. All other remaining variables are placed in wait-and-see decisions. The IBCS system aims to earn maximum revenue through appropriate offering in dayahead and intraday trading floors, as manifested by objective function (1):

$$
\begin{gathered}
\left.\Pi=\underset{\xi_{t, s}^{\mathrm{DA}}, \xi_{t, s}^{\mathrm{I}}, m_{t, s}^{\mathrm{b}}}{\operatorname{Maximize}} \mathbb{E}\left(y, \Theta_{s}\right)\right\} \\
\mathbb{E}\left\{\mathcal{F}\left(y, \Theta_{s}\right)\right\}= \\
\sum_{t \in \mathcal{T}}\left[\mathbb{E}_{S_{1}}[\underbrace{\sigma_{t, s}^{\mathrm{DA}} \xi_{t, s}^{\mathrm{DA}}}_{\Im_{1}}+\mathbb{E}_{S_{2} \mid S_{1}}[\underbrace{\sigma_{t, s}^{\mathrm{I}} \xi_{t, s}^{\mathrm{I}}}_{\Im_{2}}-\underbrace{m_{t, s}^{\mathrm{b}} C^{\mathrm{b}}}_{\Im_{3}}]]\right]
\end{gathered}
$$

where $\Pi$ and $\mathcal{F}\left(y, \Theta_{s}\right)$ are expected profit and profit function of the integrated system, respectively, and $y$ and $\Theta_{s}$ are the vectors of decision variables and stochastic parameters, respectively. $\Im_{1}$ in (2) represents the revenue of the IBCS unit from the day-ahead trading floor (first-stage, i.e., $S_{1}$ ). $\Im_{2}$ represents the intraday revenue, while $\Im_{3}$ refers to the biomass feedstock cost injected into the biomass boiler. Both $\Im_{2}$ and $\Im_{3}$ are related to the second-stage, i.e., $S_{2}$. The operational constraints of the IBCS system are given below.

$$
\begin{gathered}
\gamma_{t, s}^{\mathrm{bo}}=\eta^{\mathrm{bo}} C a^{\mathrm{b}} m_{t, s}^{\mathrm{b}} \quad \forall t, s \\
\underline{\Gamma^{\mathrm{bo}}} u_{t}^{\mathrm{bo}} \leq \gamma_{t, s}^{\mathrm{bo}} \leq \overline{\Gamma^{\mathrm{bo}}} u_{t}^{\mathrm{bo}} \quad \forall t, s, \quad \forall u_{t}^{\mathrm{bo}} \in\{0,1\} \\
0 \leq \sum_{t \in \mathcal{T}} m_{t, s}^{\mathrm{b}} \leq M^{\mathrm{b}} \quad \forall s \\
\xi_{t, s}^{\mathrm{pb}}=\xi_{t, s}^{\mathrm{k}, \mathrm{bo}}+\xi_{t, s}^{\mathrm{k}, \mathrm{f}}+\xi_{t, s}^{\mathrm{l}} \quad \forall t, s \\
\xi_{t, s}^{\mathrm{pb}}=\xi_{t, s}^{\mathrm{DA}}+\xi_{t, s}^{\mathrm{I}} \quad \forall t, s \\
0 \leq \xi_{t, s}^{\mathrm{pb}} \leq \overline{\Xi^{\mathrm{pb}}} \quad \forall t, s \\
\xi_{t, s}^{\mathrm{k}, \mathrm{bo}}=\eta^{\mathrm{pb}} \gamma_{t, s}^{\mathrm{k}, \mathrm{bo}}, \xi_{t, s}^{\mathrm{k}, \mathrm{f}}=\eta^{\mathrm{pb}} \gamma_{t, s}^{\mathrm{k}, \mathrm{f}}, \xi_{t, s}^{\mathrm{l}}=\eta^{\mathrm{pb}} \gamma_{t, s}^{\mathrm{l}} \quad \forall t, s \\
\gamma_{t, s}^{\mathrm{bo}}=\gamma_{t, s}^{\mathrm{k}, \mathrm{bo}}+\gamma_{t, s}^{\mathrm{h}, \mathrm{bo}} \quad \forall t, s \\
\gamma_{t, s}^{\mathrm{k}, \mathrm{f}}+\gamma_{t, s}^{\mathrm{h}, \mathrm{f}} \leq \bar{G}_{t}^{\mathrm{f}} \quad \forall t, s \\
\xi_{t, s}^{\mathrm{pb}}+\gamma^{\mathrm{SU}} r_{t} \quad \forall t, s, \quad \forall r_{t} \in\{0,1\} \\
\eta_{t, s}^{\mathrm{pB}} \quad \forall
\end{gathered}
$$




$$
\begin{aligned}
& \underline{\Gamma^{\mathrm{pb}}} u_{t}^{\mathrm{pb}} \leq \gamma_{t, s}^{\mathrm{pb}} \leq \overline{\Gamma^{\mathrm{pb}}} u_{t}^{\mathrm{pb}} \quad \forall t, s, \quad \forall u_{t}^{\mathrm{pb}} \in\{0,1\} \\
& r_{t}=u_{t}^{\mathrm{pb}}-u_{t-1}^{\mathrm{pb}} \quad \forall t, \quad \forall\left(r_{t}, u_{t}^{\mathrm{bo}}\right) \in\{0,1\} \\
& \gamma_{t, s}^{\mathrm{Ts}}=(1-\rho) \gamma_{t-1, s}^{\mathrm{Ts}}+\eta^{\mathrm{ch}}\left(\gamma_{t, s}^{\mathrm{h}, \mathrm{bo}}+\gamma_{t, s}^{\mathrm{h}, \mathrm{f}}\right)-\frac{\gamma_{t, s}^{\mathrm{l}}}{\eta^{\mathrm{dis}}} \quad \forall t, s \\
& \gamma_{t, s}^{\mathrm{Ts}}=\gamma_{0}^{\mathrm{Ts}} \quad \forall t=24, s \\
& \underline{\Gamma^{\mathrm{Ts}}} \leq \gamma_{t, s}^{\mathrm{Ts}} \leq \overline{\Gamma^{\mathrm{Ts}}} \quad \forall t, s \\
& \gamma_{t, s}^{\mathrm{h}, \mathrm{bo}} \leq \overline{\zeta^{\mathrm{ch}}} z_{t}^{\mathrm{ch}}, \gamma_{t, s}^{\mathrm{h}, \mathrm{f}} \leq \overline{\zeta^{\mathrm{ch}}} z_{t}^{\mathrm{ch}} \quad \forall t, s, \quad \forall z_{t}^{\mathrm{ch}} \in\{0,1\} \\
& \gamma_{t, s}^{1} \leq \overline{\zeta^{\mathrm{dis}}} z_{t}^{\mathrm{dis}} \quad \forall t, s, \quad \forall z_{t}^{\mathrm{dis}} \in\{0,1\} \\
& z_{t}^{\mathrm{ch}}+z_{t}^{\mathrm{dis}} \leq 1 \quad \forall t, \quad \forall\left(z_{t}^{\mathrm{ch}}, z_{t}^{\mathrm{dis}}\right) \in\{0,1\} \\
& u_{t}^{\mathrm{pb}} \leq z_{t}^{\mathrm{dis}} \quad \forall t, \quad \forall\left(u_{t}^{\mathrm{pb}}, z_{t}^{\mathrm{dis}}\right) \in\{0,1\} \\
& \sum_{\delta=t-t^{\text {on }, \mathrm{X}}}^{t-1} u_{\delta}^{\mathrm{X}} \geq t^{\mathrm{on}, \mathrm{X}}\left(u_{t-1}^{\mathrm{X}}-u_{t}^{\mathrm{X}}\right) \quad \forall t, \mathrm{X}=[\mathrm{pb}, \mathrm{bo}] \\
& \sum_{\delta=t-t^{\text {off }, \mathrm{X}}}^{t-1}\left(1-u_{\delta}^{\mathrm{X}}\right) \geq t^{\mathrm{off}, \mathrm{X}}\left(u_{t}^{\mathrm{X}}-u_{t-1}^{\mathrm{X}}\right) \quad \forall t, \mathrm{X}=[\mathrm{pb}, \mathrm{bo}] \\
& \xi_{t-1, s}^{\mathrm{pb}} \leq \xi_{t, s}^{\mathrm{pb}}+\Delta^{\text {down }} \quad \forall t, s \\
& \xi_{t, s}^{\mathrm{pb}} \leq \xi_{t-1, s}^{\mathrm{pb}}+\Delta^{\mathrm{up}} \quad \forall t, s \\
& 0 \leq \xi_{t, s}^{\mathrm{I}} \leq \Upsilon \xi_{t, s}^{\mathrm{D}} \quad \forall t, s \\
& \xi_{t, s}^{\mathrm{DA}} \geq \xi_{t, s^{\prime}}^{\mathrm{DA}} \text { if } \sigma_{t, s}^{\mathrm{DA}} \geq \sigma_{t, s^{\prime}}^{\mathrm{DA}} \quad \forall t, s, s^{\prime} \\
& \xi_{t, s}^{\mathrm{DA}}=\xi_{t, s^{\prime}}^{\mathrm{DA}} \text { if } \sigma_{t, s}^{\mathrm{DA}}=\sigma_{t, s^{\prime}}^{\mathrm{DA}} \quad \forall t, s, s^{\prime} \\
& \xi_{t, s}^{\mathrm{I}}=\xi_{t, s^{\prime}}^{\mathrm{I}} \text { if } \sigma_{t, s}^{\mathrm{DA}}=\sigma_{t, s^{\prime}}^{\mathrm{DA}} \quad \forall t, s, s^{\prime}
\end{aligned}
$$

Equation (3) simulates the thermal energy produced by the biomass boiler, while constraint (4) restricts it within acceptable boundaries. Inter-temporal constraint (5) imposes the limit of the injected feedstock to the biomass boiler during the scheduling horizon. Equations (6) and (7) denote the electric power generated by the power block, whereas constraint (8) restricts it within tolerable bounds. Equation (9) defines the electric power generated via thermal energy transfer from the biomass boiler, solar field, or thermal storage. The thermal energy from the biomass boiler and solar field can be transferred to the power block or thermal storage, as expressed in (10) and (11). Equation (12) represents the power block's input thermal energy, and constraint (13) restricts this variable within acceptable boundaries. Equation (14) defines the start-up state of the power block. The energy level of the thermal storage at each hour is calculated via (15). Moreover, constraint (16) enforces that the energy level at the final hour of the scheduling horizon must be equal to its initial energy. The energy level calculated in (15) must be kept within the tolerable bounds, as stated in (17). Maximum charging and discharging rates of the thermal storage are imposed through (18) and (19). Concurrent charging and discharging of the thermal storage is prevented by (20). Constraint 21) states that thermal storage can be discharged if and only if the power block is running. Minimum up- and down-times limits for the biomass boiler and power block are enforced in (22) and 23. The power block's ramp-down and ramp-up constraints are imposed by 24) and 25. Constraint 26 restricts the intraday dispatch power to a portion of the day-ahead power dispatch. The day-ahead power dispatch must be ascending, as denoted in (27). Nonanticipativity restriction of day-ahead and intraday dispatch powers is enforced by (28) and (29). In preceding operational constraints, constraints (14), 20)-(23), 27), and 29) are first-stage constraints, whereas the remaining constraints act as linking constraints, encompassing both firststage and second-stage variables. It is essential to remark that in the above model, the correlation between day-ahead and intraday price scenarios is captured by defining a new parameter $\varkappa_{t, s}$ serving as the difference between intraday and day-ahead prices (see Appendix A). Therefore, the parameter $\sigma_{t, s}^{\mathrm{I}}$ should be replaced by $\varkappa_{t, s}+\sigma_{t, s}^{\mathrm{D}}$ to account for the correlation between day-ahead and intraday price scenarios [19].

\section{INCORPORATING RISK EVALUATION INDICES}

In this section, the inclusion of CVaR and IGDT management risk indices in the stochastic formulation given in the preceding section is discussed. First, the incorporation of the risk evaluation index for uncertain stochastic inputs $\left(\sigma_{t, s}^{\mathrm{DA}}, \sigma_{t, s}^{\mathrm{I}}\right)$, i.e., $\mathrm{CVaR}$, is presented in subsection IV-A Subsequently, the consolidation of the IGDT technique for uncertain thermal power of solar field $\left(\tilde{G}_{t}^{\mathrm{f}}\right)$ is discussed in subsection IV-B.

\section{A. Incorporating CVaR Risk Evaluation Index}

To incorporate the CVaR criterion into the stochastic formulation provided in Section III, the objective function 30, subject to constraints (31)-(33) must be optimized [19]. It is worth mentioning that $\Lambda$ in 30 is a non-negative number reflecting the risk-averse degree of the decision-maker, and the resulting formulation remains mixed-integer linear.

$$
\begin{gathered}
\underset{\xi_{t, s}^{\mathrm{DA}}, \xi_{t, s}^{\mathrm{I}}, m_{t, s}^{\mathrm{b}}, \Psi, Q_{s}}{\operatorname{Maximize}} \mathbb{E}\left\{\mathcal{F}\left(y, \Theta_{s}\right)\right\}+\left(\Lambda \times \mathrm{CVaR}_{\kappa}\right) \\
\mathrm{CVaR}_{\kappa}=\Psi-\frac{1}{1-\kappa} \sum_{s \in \mathcal{S}} \pi_{s} Q_{s} \\
\Psi-\sum_{t \in \mathcal{T}} \sigma_{t, s}^{\mathrm{DA}} \xi_{t, s}^{\mathrm{DA}}+\sigma_{t, s}^{\mathrm{I}} \xi_{t, s}^{\mathrm{I}}-m_{t, s}^{\mathrm{b}} C^{\mathrm{b}} \leq Q_{s} \quad \forall s \\
\text { Constraints } 2]-\quad Q_{s} \geq 0 \quad \forall s
\end{gathered}
$$

\section{B. Incorporating IGDT Risk Evaluation Index}

Accounting for the uncertainty of the thermal power output from the solar field, the IGDT approach is employed here. Under such an approach, the IBCS system can take both riskaverse and risk-seeker actions against solar-related uncertainty, both leading to different formulations as explained hereunder. Using the envelope-bound IGDT pattern [20], the uncertainty set of thermal power output from the solar field $\mathcal{R}\left(\alpha, \bar{G}_{t}^{\mathrm{f}}\right)$ is mathematically expressed as: 


$$
\mathcal{R}\left(\alpha, \bar{G}_{t}^{\mathrm{f}}\right)=\left\{\tilde{G}_{t}^{\mathrm{f}}:\left|\tilde{G}_{t}^{\mathrm{f}}-\bar{G}_{t}^{\mathrm{f}}\right| \leq \alpha \bar{G}_{t}^{\mathrm{f}}\right\} \quad \alpha \geq 0, \forall t
$$

where $\alpha$ is the uncertainty radius (or horizon), and $\bar{G}_{t}^{\mathrm{f}}$ and $\tilde{G}_{t}^{\mathrm{f}}$ are the forecasted and uncertain thermal power output from the solar field, respectively. In line with this approach, the risk-averse and risk-seeker day-ahead and intraday dispatch models facing the solar-related uncertainty are developed in the following.

\section{1) Risk-Averse IGDT model}

The risk-averse IGDT pattern for day-ahead and intraday dispatch of the IBCS system facing solar-related uncertainty is formed as the following bi-level programming problem.

$$
\begin{aligned}
& \underset{\left[\xi_{t, s}^{\mathrm{DA}}, \xi_{t, s}^{\mathrm{I}}, m_{t, s}^{\mathrm{b}}\right] \cup \tilde{G}_{t}^{\mathrm{f}}}{\operatorname{Maximize}}\left\{\alpha^{\text {scheme }_{1}}\right\} \\
& \text { s.t. }\left[\underset{\tilde{G}_{t}^{f} \in \mathcal{R}\left(\alpha, \bar{G}_{t}^{f}\right)}{\operatorname{Minimize}} \mathbb{E}\left\{\mathcal{F}\left(y, \Theta_{s}\right)\right\}\right] \geq\left(1-\beta^{\text {scheme }_{1}}\right) \Pi_{0} \\
& \gamma_{t, s}^{\mathrm{k}, \mathrm{f}}+\gamma_{t, s}^{\mathrm{h}, \mathrm{f}} \leq \tilde{G}_{t}^{\mathrm{f}} \quad \forall t, s \\
& \text { Constraints (2) - 10, (12) - 29) }
\end{aligned}
$$

where (35) and (36) are the objective functions of the upper and lower levels, respectively. The aim of the upper level is to maximize the uncertainty radius $\alpha^{\text {scheme }_{1}}$ in such a way that the objective of the lower level is met. Since the lower level problem is a linear programming problem, the minimum value of the lower level problem is acquired when $\tilde{G}_{t}^{\mathrm{f}}$ takes its lower bound. It has to be noted that $\Pi_{0}$ stands for the expected profit of the integrated system corresponding to the optimized value obtained by the optimization problem (1)- 29). In this regard, the single-level counterpart for the risk-averse IBCS system is established as follows:

$$
\begin{array}{cc}
\underset{\left[\xi_{t, s}^{\mathrm{DA}}, \xi_{t, s}^{\mathrm{I}}, m_{t, s}^{\mathrm{b}}\right] \cup \tilde{G}_{t}^{\mathrm{f}}}{\text { Maximize }}\left\{\alpha^{\text {scheme }_{1}}\right\} \\
\text { s.t. } \quad \mathbb{E}\left\{\mathcal{F}\left(y, \Theta_{s}\right)\right\} \geq\left(1-\beta^{\text {scheme }_{1}}\right) \Pi_{0} \\
\gamma_{t, s}^{\mathrm{k}, \mathrm{f}}+\gamma_{t, s}^{\mathrm{h}, \mathrm{f}} \leq\left(1-\alpha^{\text {scheme }_{1}}\right) \bar{G}_{t}^{\mathrm{f}} \quad \forall t, s \\
\text { Constraints }(2)-10,(12)-29
\end{array}
$$

\section{Risk-Seeker IGDT model}

The risk-seeker IGDT paradigm for day-ahead and intraday dispatch of the IBCS system encountering the uncertainty of the thermal power from the solar field is formed as the following bi-level programming problem.

$$
\begin{gathered}
\underset{\substack{\text { Minimize } \\
\left[\xi_{t, s}^{\mathrm{A}}, \xi_{t, s}^{\mathrm{I}}, m_{t, s}^{\mathrm{b}}\right] \cup \tilde{G}_{t}^{\mathrm{f}}}}{ }\left\{\alpha^{\text {scheme }_{2}}\right\} \\
\text { s.t. } \quad\left[\underset{\tilde{G}_{t}^{\mathrm{f}} \in \mathcal{R}\left(\alpha, \bar{G}_{t}^{\mathrm{f}}\right)}{\operatorname{Maximize}} \mathbb{E}\left\{\mathcal{F}\left(y, \Theta_{s}\right)\right\}\right] \geq\left(1+\beta^{\text {scheme }_{2}}\right) \Pi_{0} \\
\gamma_{t, s}^{\mathrm{k}, \mathrm{f}}+\gamma_{t, s}^{\mathrm{h}, \mathrm{f}} \leq \tilde{G}_{t}^{\mathrm{f}} \quad \forall t, s
\end{gathered}
$$

Constraints 22 - 10, (12) - 29) where (43) and (44) are the objective functions of the upper and lower levels, respectively. The purpose of the upper level is to minimize the uncertainty radius $\alpha^{\text {scheme }}$ in a manner that the objective of the lower level is fulfilled. As the lower level problem is a linear optimization problem, the maximum value of the lower level problem is achieved if $\tilde{G}_{t}^{\mathrm{f}}$ takes its upper bound. In this vein, the single-level counterpart for the riskseeker IBCS system is mathematically formulated as follows:

$$
\begin{aligned}
& \underset{\left[\xi_{t, s}^{\mathrm{DA}}, \xi_{t, s}^{\mathrm{I}}, m_{t, s}^{\mathrm{b}}\right] \cup \tilde{G}_{t}^{\mathrm{f}}}{\operatorname{Minimize}}\left\{\alpha^{\text {scheme }_{2}}\right\} \\
& \text { s.t. } \mathbb{E}\left\{\mathcal{F}\left(y, \Theta_{s}\right)\right\} \geq\left(1+\beta^{\text {scheme }_{2}}\right) \Pi_{0} \\
& \gamma_{t, s}^{\mathrm{k}, \mathrm{f}}+\gamma_{t, s}^{\mathrm{h}, \mathrm{f}} \leq\left(1+\alpha^{\mathrm{scheme}_{2}}\right) \bar{G}_{t}^{\mathrm{f}} \quad \forall t, s \\
& \left(1+\alpha^{\text {scheme }_{2}}\right) \bar{G}_{t}^{\mathrm{f}} \leq \overline{G G} \quad \forall t \\
& \text { Constraints (2) - (10), (12) - 29) }
\end{aligned}
$$

When seeking opportunistic circumstances for the thermal power output of the solar field, constraint (50) must be included in the methodology to limit this parameter within the maximum available capacity. This constraint was neglected in [13].

\section{Proposed Multi-Objective CVaR-IGDT ALGORITHM}

This section is dedicated to the procedure of managing CVaR and IGDT risk criteria simultaneously and deriving the optimal scheduling strategy among several generated strategies. By setting up the proposed hybrid risk management model, the IBCS system could adopt the following riskcontrolling-schemes:

- Risk-Controlling Scheme 1: The decision-maker takes risk-averse actions against both stochastic and IGDT inputs.

- Risk-Controlling Scheme 2: The decision-maker takes risk-averse actions against stochastic inputs while seeking opportunistic situations concerning the IGDT parameter.

It should be noted that there is no specific relation between IGDT and CVaR risk criteria, especially in the mathematical modeling of each risk criterion. The algorithm of adopting day-ahead and intraday dispatch strategies based upon riskcontrolling scheme 1 is summarized in Algorithm 11. To avoid repetition, the algorithm for risk-controlling scheme 2 is provided in Appendix B. Note that the proposed CVaR-IGDT model is generic and can be applied to other scheduling problems with different sources of uncertainty (jointly involving market-related and weather-related parameters).

Aside from extracting diverse risk-controlling strategies using the proposed CVaR-IGDT procedure, it is of great importance to derive the final optimal strategy for the IBCS system. To this end, a posteriori approach [20] based on the out-of-sample assessment [23] is conducted in this paper. The algorithm of a posteriori approach is delineated in Algorithm 2 


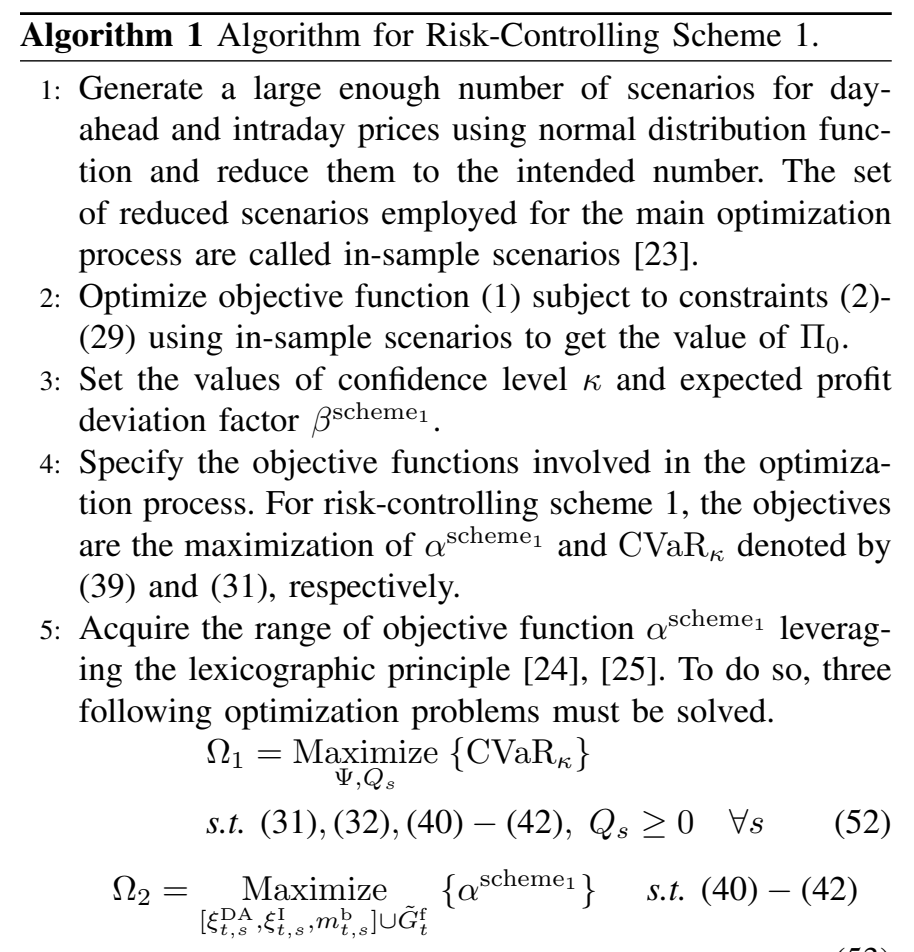

$$
\begin{array}{r}
\Omega_{3}=\underset{\xi_{t, s}^{\mathrm{DA}}, \xi_{t, s}^{\mathrm{I}}, m_{t, s}^{\mathrm{b}}}{\operatorname{Maximize}}\left\{\alpha^{\text {scheme }_{1}} \mid \mathrm{CVaR}_{\kappa}=\Omega_{1}\right\} \\
\text { s.t. 31, 32, 40) }- \text { 42, }, Q_{s} \geq 0 \quad \forall s
\end{array}
$$

Then, the range of $\alpha^{\text {scheme }_{1}}$ is equal to $\Omega_{3}-\Omega_{2}$.

6: Generate different optimal Pareto strategies for objectives specified in step 4 leveraging $\epsilon$-constraint method. This is accomplished by means of optimizing the following problem.

$$
\begin{array}{ll}
\underset{\xi_{t, s}^{\mathrm{DA}}, \xi_{t, s}^{\mathrm{I}}, m_{t, s}^{\mathrm{b}}, \Psi, Q_{s}, \vartheta}{\text { Maximize }} & \mathrm{CVaR}_{\kappa}-\frac{\vartheta}{\Omega_{3}-\Omega_{2}} \\
\text { s.t. } & \alpha^{\text {scheme }_{1}}-\vartheta=N_{\epsilon} \\
& N_{\epsilon}=\Omega_{2}+\left(\frac{\Omega_{3}-\Omega_{2}}{p}\right) \times \epsilon, \quad \epsilon=0,1, \ldots, p \\
& \text { 31, 32, }, 407-42, Q_{s} \geq 0 \quad \forall s, \quad \vartheta \geq 0
\end{array}
$$

In (55), $\vartheta$ refers to an extra variable. By selecting the desired value for $p, p+1$ Pareto strategies will be obtained.

\section{Results}

In this paper, a 100-MW IBCS system created by integrating 50-MW biomass and concentrated solar units is considered to assess the benefits of the proposed structure. The parameters of the intended IBCS system, along with the set of data used for the scenario generation of stochastic parameters $\left(\sigma_{t, s}^{\mathrm{DA}}, \sigma_{t, s}^{\mathrm{I}}\right)$, are presented in Appendices A and C. To generate large enough scenarios for stochastic inputs, the normal distribution function is used. To relieve computational burden, the forward reduction mechanism is exploited to decrease the set of scenarios produced for each stochastic input to twenty samples, known as in-sample scenarios. The mathematical programming model has been coded in GAMS and solved using CPLEX as the solver. All simulations are
Algorithm 2 Algorithm for a posteriori approach.

1: Generate an adequate number of scenarios for both stochastic and IGDT inputs. These scenarios are named out-of-sample scenarios. It is worth emphasizing that the set of generated scenarios is totally different from the in-sample scenarios utilized for the main optimization process.

2: For each Pareto strategy obtained from risk-controlling schemes, extract the first-stage decision variables.

3: Optimize the deterministic model of the second-stage problem (1)-29) for each out-of-sample scenario by fixing the first-stage variables to the extracted values in the previous step.

4: Reiterate step 3 for all out-of-sample scenarios and calculate the expected profit of the IBCS system.

5: Reiterate steps 2 to 4 for all Pareto strategies and report the expected profit values obtained from step 4.

6: Select the Pareto strategy with the highest value of expected profit reported in the previous step as the optimal dispatch plan.

conducted on a Laptop with an Intel Core i5 processor and 8GB DDR3 memory. Note that the model is mixed-integer linear programming, and the value of $\kappa$ is set to 0.95 in all analyses and studies [12], [19].

To assess various aspects of this work, three different studies are carried out in subsections VI-A to VI-C. Subsection VI-A investigates the profitability of the suggested integrated dispatch framework compared to the individual one. Subsection VI-B analyzes the effectiveness of the proposed risk management architecture in offering various optimal dispatch strategies. Subsection VI-C explores the performance of the proposed CVaR-IGDT model versus conventional approaches.

\section{A. Profitability Analysis of the Integrated Dispatch Model}

This analysis aims to investigate the economic prosperity of integrating biomass and concentrated solar units versus individually dispatching them. In this analysis, the solar field's thermal power is considered deterministic, while stochastic inputs are present in the model. The configuration of the IBCS system is given in Fig. 1. while the configuration of individual biomass and concentrated solar units is shown in Fig. 2. The parameters of the IBCS system are provided in Appendix A. For individual biomass and concentrated solar units, the technical characteristics of the biomass boiler, solar field, and thermal energy storage are the same as the ones of the IBCS system. The parameters of the power block in individual generating units (Fig. 2) has the following differences with the IBCS system (Fig. 1):

- The electric power capacity of power blocks in individual biomass and concentrated solar units is 50-MW. Note that this parameter is equal to $100-\mathrm{MW}$ for the IBCS system. Therefore, minimum and maximum thermal power limits of power blocks in individual generating units $\left(\underline{\Gamma^{\mathrm{pb}}}, \overline{\Gamma^{\mathrm{pb}}}\right)$ is $50-\mathrm{MW}$ and $125-\mathrm{MW}$, respectively.

- The ramp-down and ramp-up limits of power blocks are typically equal to $40 \%$ of their electric power capacity 


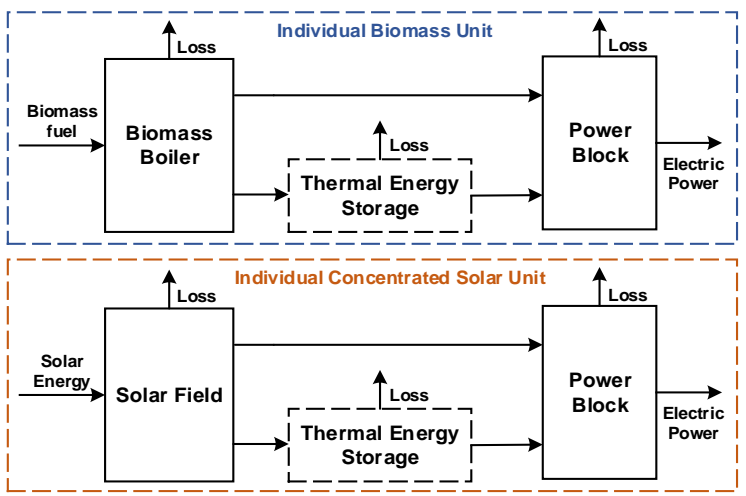

Fig. 2: Configuration of individual biomass and concentrated solar units.

TABLE II: Added-value of the integrated dispatch model in terms of profit and CVaR for each Pareto strategy [€].

\begin{tabular}{|c|c|c|c|c|c|}
\hline & P1 & P2 & P3 & P4 & P5 \\
\hline Profit & $1,875.945$ & $1,875.695$ & $1,946.934$ & 1946.141 & $1,945.338$ \\
\hline CVaR & $2,682.902$ & $2,641.743$ & $2,600.584$ & 2559.426 & $2,518.267$ \\
\hline & P6 & P7 & P8 & P9 & P10 \\
\hline Profit & $1,944.366$ & $1,938.353$ & $1,909.993$ & $1,901.691$ & $1,945.386$ \\
\hline CVaR & $2,477.109$ & $2,435.949$ & $2,394.791$ & $2,353.632$ & $2,312.474$ \\
\hline
\end{tabular}

[9]. Thus, $\Delta^{\text {down }}$ and $\Delta^{\text {up }}$ for individual biomass and concentrated solar units are set to $20 \mathrm{MW} / \mathrm{hr}$.

For the sake of a fair analysis and comparison, other parameters of power blocks in the individual dispatch model $\left(t^{\mathrm{off}, \mathrm{pb}}, t^{\mathrm{on}, \mathrm{pb}}, \gamma^{\mathrm{SU}}\right.$, and $\left.\eta^{\mathrm{pb}}\right)$ are considered similar to the IBCS system.

We provide a profit-CVaR study in this subsection to not only examine the profitability of the integrated dispatch model but also to assess the effect of this integration on the present risk index, namely, CVaR. Hence, this analysis concerns a multi-objective optimization model in which equations (1D) and (31) are the objective functions, while the optimization constraints include constraints (2)-(29) and (32)-(33). This multi-objective optimization is solved using lexicographic plus $\epsilon$-constraint, similar to the procedure described in Algorithm 1 . We set the input parameters of the multi-objective optimization model such that ten Pareto strategies are derived for good enough resolution. Fig. 3 shows the comparison results of the integrated and individual dispatch models. In this figure, P1, P2 , .., P10 refer to the Pareto strategies obtained from the multi-objective optimization discussed earlier. From Fig. 3. we observe that the proposed integrated dispatch framework offers greater profit and $\mathrm{CVaR}$ versus the individual dispatch model. A cautionary note is that a larger $\mathrm{CVaR}$ implies lower risk exposure. The added-value of the integrated dispatch model in terms of profit and CVaR for different Pareto strategies denoted in Fig. 3 is reported in Table $\Pi$. As Table $\Pi$ shows, the addedvalue of profit for the IBCS system is remarkable. It can be seen that the added value of profit would be at least $€ 1875$ daily, considering all Pareto strategies. Also, it is observed that the integrated dispatch model can significantly assist in reaching lower-level risk strategies.

To further demonstrate the difference between individual
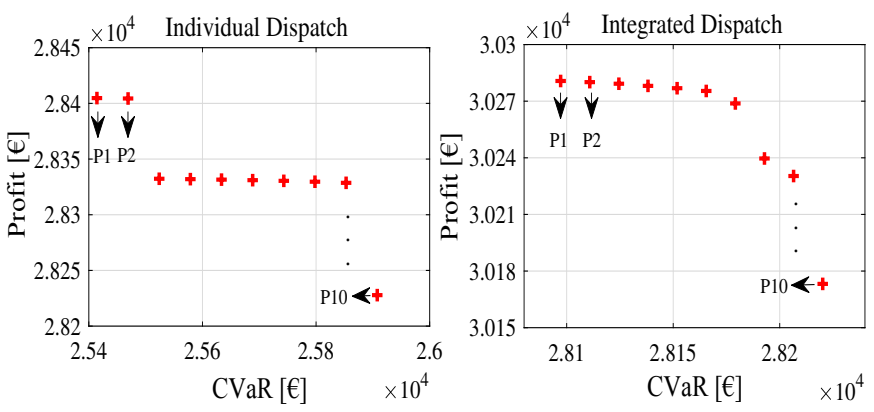

Fig. 3: Profit versus CVaR in integrated and individual dispatch models.

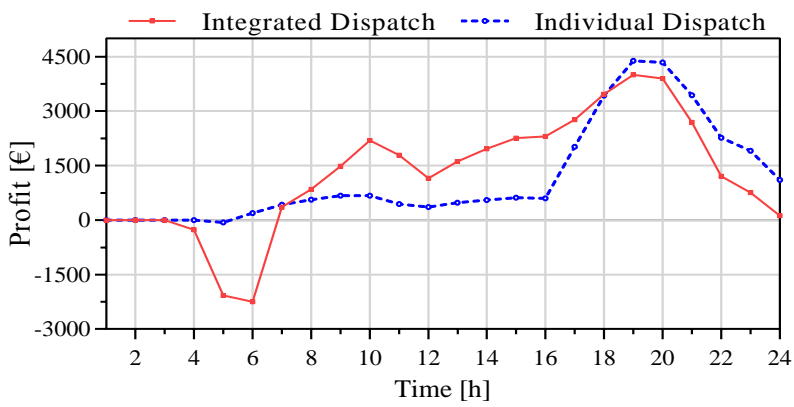

Fig. 4: Hourly profit of individual and integrated dispatch models in Pareto strategy P1.

and integrated dispatch strategies, Figs. 4, 5, and 6 are provided. The presented results pertain to Pareto strategy P1 with the greatest profit among all other Pareto strategies, as specified in Fig. 3 and Table III Fig. 4 compares the hourly profit of integrated and individual dispatches. This figure clearly shows how integration leads to higher profits of the IBCS system. Contrary to the individual dispatch which aims to gain more profit in the evening, the IBCS system allows attaining higher profit from late morning to late afternoon. The reason comes from the fact the integrated dispatch charges the thermal energy storage during hours 4-6 (negative profit in Fig. 44 for later use in the mentioned period (late morning to late afternoon). It is worth noting that negative profit in Fig. 4 arises from producing thermal energy by the biomass boiler and transferring it to the thermal energy storage. It can be seen that negative profit is not observed in the individual dispatch, meaning that such an approach is not cost-effective for individually dispatching biomass and concentrated solar units.

To better illustrate the differences between integrated and individual systems, the optimal dispatch of these systems at two representative hours (10 and 22) is depicted in Figs. 5 and 6 As Fig. 5 shows, at hour 10 of the individual dispatch, 26.7ton biomass is burned at the biomass boiler to directly generate $38.9 \mathrm{MW}$ power, while the whole solar energy is stored at the thermal energy storage for later use in the evening. At the same hour, a lower mass of biomass (25.4-ton) is burned at the biomass boiler to directly transfer $56.2 \mathrm{MW}$ thermal energy to the power block in the integrated dispatch. In contrast to the individual model, in the integrated dispatch, the whole solar energy in the integrated dispatch and $0.7 \mathrm{MW}$ thermal energy 


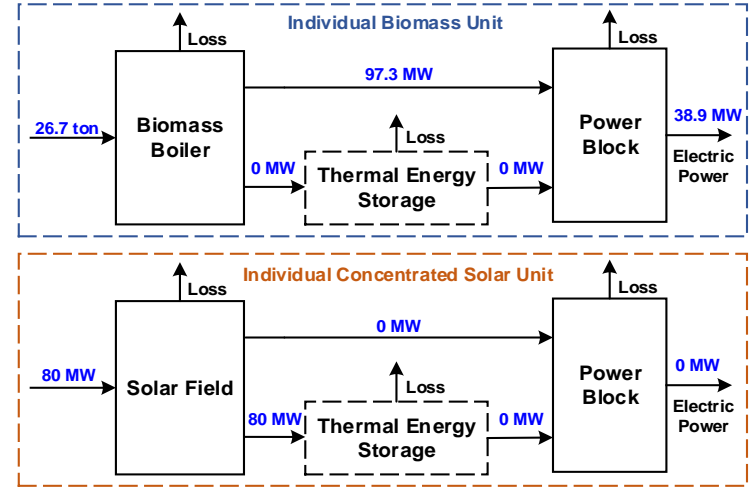

(a) Individual dispatch model.

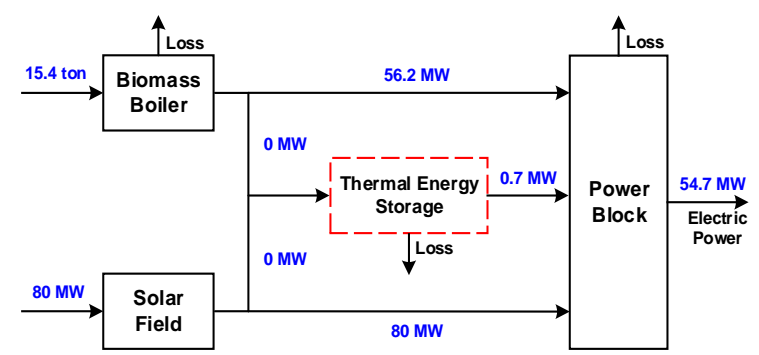

(b) Integrated dispatch model.

Fig. 5: Optimal dispatch of individual and integrated dispatch models in Pareto strategy P1 at hour 10.

from the thermal energy storage are transferred to the power block, resulting in a 54.7 MW power output. This shows that the integrated dispatch results in greater output power (profit) than the individual one at hour 10, as shown in Fig. 4 Note that the integrated dispatch does not need the solar energy to charge the thermal energy storage for later use, as done in the individual model, since all required energy for the charging process is provided by the biomass boiler during hours 4-6 (as discussed earlier).

The reason behind the greater profit of the individual dispatch at hour 22 can be explicitly seen in Fig. 6. The greater output electric power of the individual dispatch at this hour arises from the higher rate of discharging power from the thermal energy storage. This leads to the conclusion that in the individual dispatch, the stored energy in the thermal energy storage is exploited in the evening. In contrast, in the integrated model, the stored energy is exploited over a longer period, which leads to an overall higher profit of the integrated dispatch.

\section{B. Analysis of the proposed CVaR-IGDT model}

In this part of the study, we focus on the functioning of the proposed risk-controlling schemes described in Algorithm 1 and Algorithm 3 . The values of $\beta^{\text {scheme }_{1}}$ and $\beta^{\text {scheme }_{2}}$ in these algorithms are set to 0.09 , meaning that the value of expected profit for different Pareto strategies in riskcontrolling scheme $1 / 2$ must be less/ greater than or equal to the value of $\left(1-\beta^{\text {scheme }_{1}}\right) \Pi_{0} /\left(1+\beta^{\text {scheme }_{2}}\right) \Pi_{0}$. Further, $p$ in both algorithms is set to nine to achieve ten different Pareto Strategies. First, the performance of the proposed CVaR-IGDT model with the one suggested in [13] is compared and then various aspects of the proposed model are examined. For an

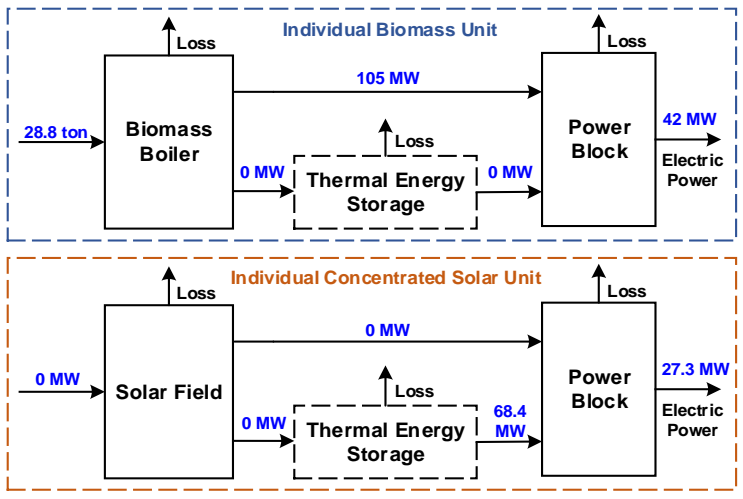

(a) Individual dispatch model.

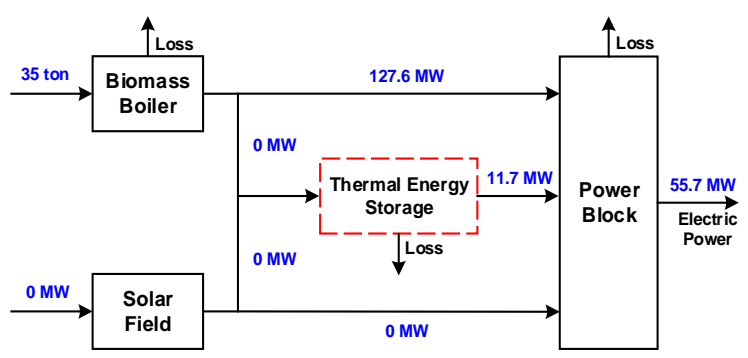

(b) Integrated dispatch model.

Fig. 6: Optimal dispatch of individual and integrated dispatch models in Pareto strategy P1 at hour 22.

efficient comparison, the input parameters of model [13] are chosen in a similar fashion to that of [13] to obtain ten different Pareto strategies. The comparison between the obtained Pareto strategies in this paper and the one suggested in [13] for both risk-controlling schemes is given in Fig. 7 Note that in all simulations, the value of $\beta^{\text {scheme }_{1}}$ and $\beta^{\text {scheme }_{2}}$ is set to 0.09. From Fig. 7, it can be seen that: (1) the proposed model can build Pareto strategies that are regularly distanced solutions, in contrast with Ref. [13]; (2) the proposed model covers a wider range of values for all three variables presented in Fig. 7, while Ref. [13] can only cover a limited area of values as a result of not directly handling the original CVaR index. Besides, as shown in Table III], the quality of Pareto strategies obtained by the proposed model is higher than the one of Ref. [13]. It should be noted that the results reported in Table III pertain to Pareto strategies with an equal value of profit. It is shown that in both risk-controlling schemes, the proposed model holds a higher CVaR value compared to Ref. [13], implying a lower risk exposure. Moreover, for risk-controlling scheme 1, the proposed model attains a lower uncertainty radius in comparison to Ref. [13], meaning that it reaches a specific profit in a lower uncertainty radius of the IGDT parameter.

Here, we specifically analyze the performance of the proposed CVaR-IGDT model. By executing Algorithms 1 and 3 , the set of Pareto strategies for risk-controlling schemes 1 and 2 are obtained, as shown in Fig. 8. Note that the set of Pareto strategies in Fig. 8 are the same as the Pareto strategies depicted in Fig. 7 As Fig. 8 shows in greater detail, the proposed CVaR-IGDT model is proficient in generating regularly distanced strategies. The trend of Pareto strategies 


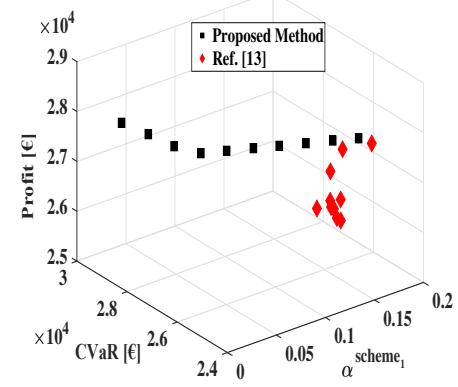

(a) Risk-Controlling Scheme 1.

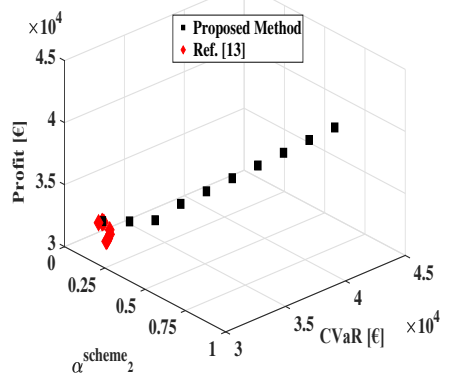

(b) Risk-Controlling Scheme 2.

Fig. 7: Comparison between the obtained Pareto strategies in the proposed model and the model of Ref. [13].

TABLE III: Comparison between Pareto strategies with an equal value of profit in the proposed model and the model of Ref. [13].

\begin{tabular}{|c|c|c|c|c|}
\hline \multirow[t]{2}{*}{ Model } & \multicolumn{2}{|c|}{$\begin{array}{c}\text { Risk-Controlling } \\
\text { Scheme } 1 \\
\text { Profit= } € 27555.434\end{array}$} & \multicolumn{2}{|c|}{$\begin{array}{c}\text { Risk-Controlling } \\
\text { Scheme } 2 \\
\text { Profit= } € 33005.960\end{array}$} \\
\hline & $\alpha^{\text {scheme }_{1}}$ & CVaR $[€]$ & $\alpha^{\text {scheme }_{2}}$ & CVaR $[€]$ \\
\hline Ref. |13| & 0.179 & 25243.414 & 0.181 & 30404.939 \\
\hline Proposed Method & 0.176 & 25649.315 & 0.181 & 30726.660 \\
\hline
\end{tabular}

in risk-controlling scheme 1 shows a continuous reduction for CVaR by increasing the value of uncertainty radius $\left(\alpha^{\text {scheme }_{1}}\right)$. The tendency of Pareto strategies in risk-controlling scheme 2 reveals the increment of CVaR following the increase of uncertainty radius $\left(\alpha^{\text {scheme }_{2}}\right)$. In risk-controlling scheme 1 , by increasing the value of uncertainty radius $\left(\alpha^{\text {scheme }_{1}}\right)$ from 0 to 0.059 , the profit follows a downward trend. Also, in riskcontrolling scheme 2 , by increasing the value of uncertainty radius $\left(\alpha^{\text {scheme }_{2}}\right)$ from 0.258 to 0.875 , the profit follows an upward trend. Moreover, by altering the uncertainty radiuses for some Pareto strategies in both risk-controlling schemes, the profit remains fixed. For those Pareto strategies with fixed profit, it can be seen that a constant profit can be maintained while the level of risk exposure is different.

As stated earlier, the decision-maker sets the parameter $p$ to nine to achieve ten different Pareto strategies. Nevertheless, decision-makers may prefer to make decisions with respect to a larger/smaller number of Pareto strategies. It is undeniable that the larger (smaller) the number of Pareto strategies, the more (less) the computational cost. Accordingly, the value of parameter $p$ should be set based on a trade-off between the number of Pareto strategies and computational cost. Fig. 9 displays the Pareto front obtained for the multi-objective riskcontrolling scheme 1, aiming to achieve five, ten, fifteen, and twenty Pareto strategies. This figure shows that the larger the number of Pareto Strategies, the greater the coverage of the Pareto front. However, the computation time to obtain five, ten, fifteen, and twenty Pareto strategies is 239, 290, 410, and 535 seconds, revealing the rise in computational cost by increasing the number of Pareto strategies. All in all, it is up to the decision maker's preferences to select the number of Pareto strategies based on the desired computational cost.

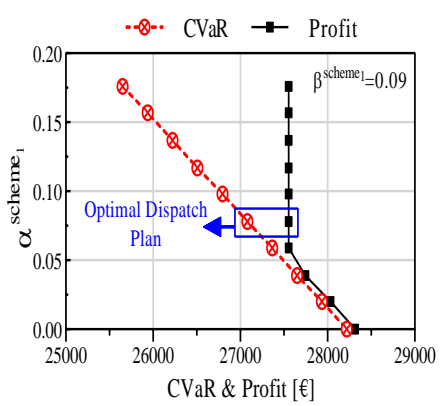

(a) Risk-controlling scheme 1 .

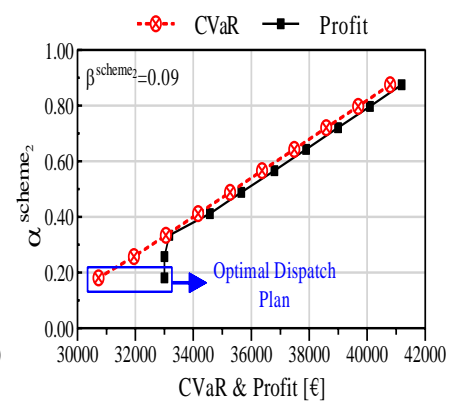

(b) Risk-controlling scheme 2 .
Fig. 8: Various Pareto strategies obtained through riskcontrolling schemes 1 and 2 .

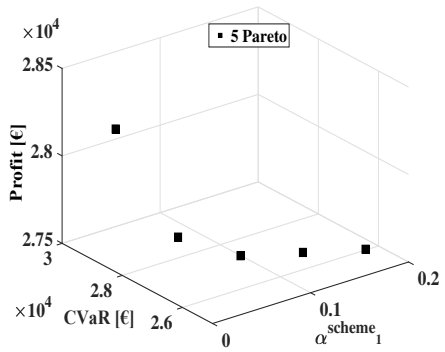

(a) Five Pareto Strategies.

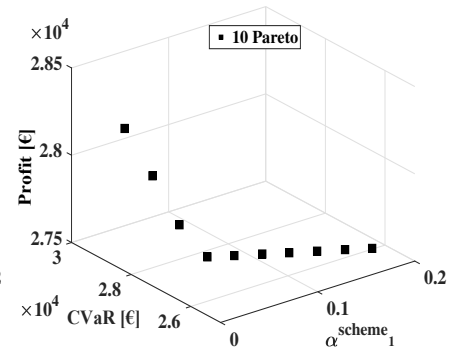

(b) Ten Pareto Strategies.

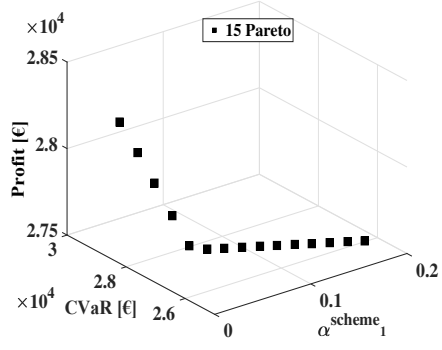

(c) Fifteen Pareto Strategies.

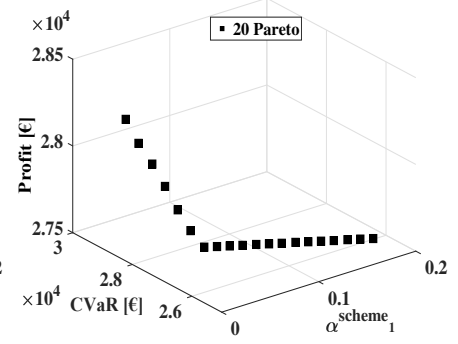

(d) Twenty Pareto Strategies.
Fig. 9: Pareto front in risk-controlling scheme 1 under different number of Pareto strategies.

To find the optimal dispatch plan, we run Algorithm 2 for the set of Pareto strategies obtained from Algorithms 1 and 3 It is essential to note that 1,000 out-of-sample scenarios are applied for a posteriori algorithm. To generate the out-ofsample scenarios for stochastic inputs, the parameters given in Appendix A are used. It has to be noticed that the set of generated scenarios for out-of-sample scenarios is quite distinct from the one used for in-sample scenarios. For the IGDT input, the standard deviation is assumed to be $10 \%$ of the forecasted value. By running Algorithm 2 the optimal dispatch plans for each risk-controlling scheme are attained, as denoted in Fig. 8 The profit, CVaR, and uncertainty radius of optimal dispatch plans are presented in Fig. 10. Since risk-controlling scheme 2 is seeking opportunistic situations in terms of solar-related uncertainty, the optimal profit in this scheme is higher than risk-controlling scheme 1 . Accordingly, a greater profit yields a higher $\mathrm{CVaR}$ in risk-controlling scheme 2 . Another point of attention is that the entire procedure of obtaining all Pareto strategies and running a posteriori algorithm took 292 and 


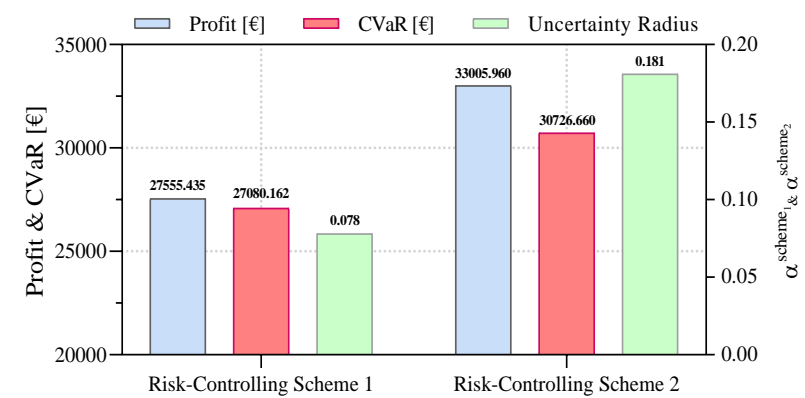

Fig. 10: Results of Optimal Dispatch Plans in Risk-Controlling Schemes 1 and 2.

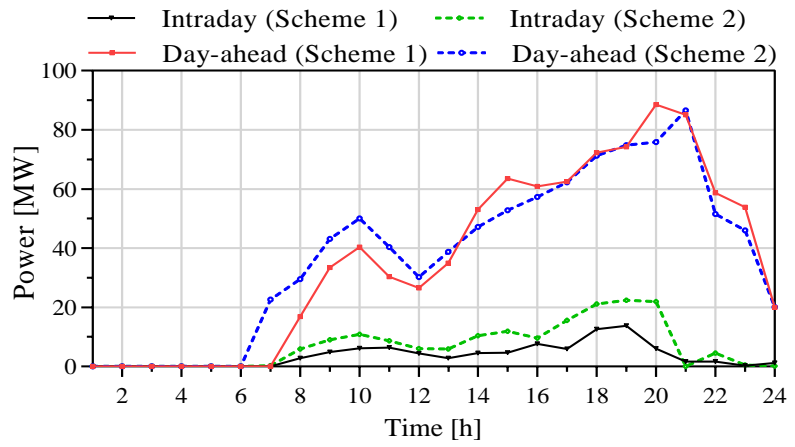

(a) Day-ahead and intraday dispatch powers.

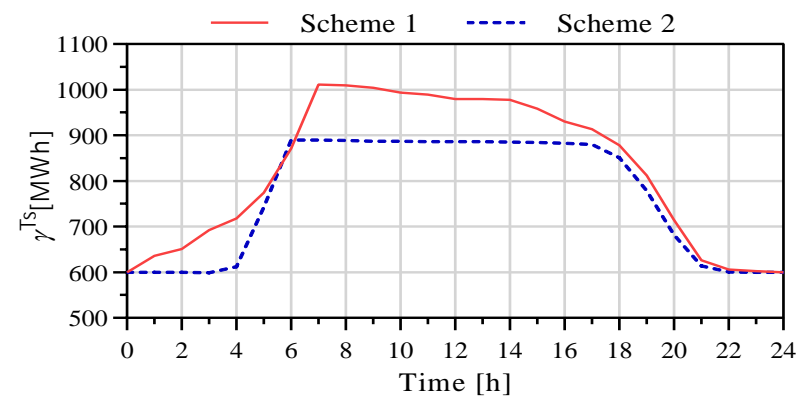

(b) Energy level of thermal storage.

Fig. 11: Optimal results in risk-controlling schemes 1 and 2.

204 seconds for risk-controlling schemes 1 and 2, respectively. Consequently, under 30 seconds of computation time for each Pareto strategy exhibits the satisfactory computational cost of the proposed methodology.

Fig. 11 displays the optimal day-ahead and intraday dispatch powers, along with the optimal pattern of energy level in the thermal storage, for both risk-controlling schemes. From Fig. 11. we observe that the day-ahead dispatch power in the second scheme is higher than the first scheme during hours 7-13, while the opposite is true for almost all remaining periods of the scheduling horizon. Also, a higher intraday dispatch power in the second scheme compared to the first scheme can be observed for almost all periods during which the IBCS system participates in the intraday trading floor. According to Fig. 11, the energy level of thermal storage in the second scheme is lower than in the first scheme. The reason is that when the IBCS system takes a risk-seeker attitude against solar-related uncertainty (risk-controlling scheme 2), more energy from the thermal storage is discharged to the
TABLE IV: Definition of In-Sample and Out-of-Sample Assessments.

\begin{tabular}{|c|c|c|}
\hline Model & In-sample assessment & aple assessment \\
\hline CVaR-IGDT & $\begin{array}{c}\text { Solve the proposed } \\
\text { CVaR-IGDT algorithm using } \\
\text { a reduced scenario set } \\
\text { named in-sample scenarios, } \\
\text { as denoted in the first step } \\
\text { of Algorithm } 1 \text { or } 3\end{array}$ & \multirow{3}{*}{$\begin{array}{l}\text { Extract the first-stage } \\
\text { decision variables } \\
\text { (see Table I from the } \\
\text { in-sample assessment. Then, } \\
\text { solve the deterministic } \\
\text { model of the second-stage } \\
\text { problem } 1 \text { - } 29 \text { for each } \\
\text { out-of-sample scenario by } \\
\text { fixing the first-stage decision } \\
\text { variables to the extracted } \\
\text { values from the in-sample } \\
\text { assessment. }\end{array}$} \\
\hline Deterministic & $\begin{array}{l}\text { Solve the deterministic } \\
\text { model of } 11-29 \\
\text { by replacing the uncertain } \\
\text { parameters with their } \\
\text { mean values. }\end{array}$ & \\
\hline Full stochastic & $\begin{array}{l}\text { Solve two-stage stochastic } \\
\text { model }[1]-[29] \text { using } \\
\text { in-sample scenarios and } \\
\text { by replacing the IGDT } \\
\text { parameter with a } \\
\text { reduced scenario set. }\end{array}$ & \\
\hline
\end{tabular}

power block to boost the whole system's revenue. The higher discharge power from thermal storage is due to overestimating the output thermal power of the solar field in opportunistic circumstances.

\section{Performance of the proposed CVaR-IGDT model}

The performance of the proposed CVaR-IGDT riskcontrolling method against conventional approaches, namely, deterministic and full stochastic methods, is evaluated in this subsection. To this end, in-sample and out-of-sample assessments according to Table [V] are carried out [23]. It has to be noted that we use the mean values of uncertain inputs for the deterministic approach. In contrast, for the full stochastic approach, we rely on twenty scenarios resulting from a scenario generation and reduction process (similar to step 1 in Algorithm 11) to characterize solar-related uncertainty, whereas the day-ahead and intraday scenarios are the same samples used in the proposed CVaR-IGDT model. For the out-of-sample assessment, 1000 samples are employed. It is worth noting that for both risk-controlling schemes under the CVaR-IGDT model, we consider all values $\beta^{\text {scheme }_{1,2}}=$ $0.01,0.02,0.03, \ldots, 0.15$. The results of in-sample and outof-sample assessments for different approaches are reported in Fig. 12. The results show that the proposed CVaR-IGDT model in both in-sample and out-of-sample assessments leads to a higher expected profit, manifesting the superior performance of this model versus deterministic and full stochastic approaches. Although the profit increment in the in-sample analysis is more significant, the profit increase in the out-ofsample assessment is also considerable. Based on the results, it can be estimated that by leveraging the proposed CVaR-IGDT model, a substantial annual added-value in the expected profit of the IBCS system will be obtained.

\section{CONCLUSION}

This paper presented a day-ahead and intraday dispatch model for an IBCS system. The objective was to present a suitable coupling scheme for large-scale biomass and concentrated solar power systems and explore the profitability of 


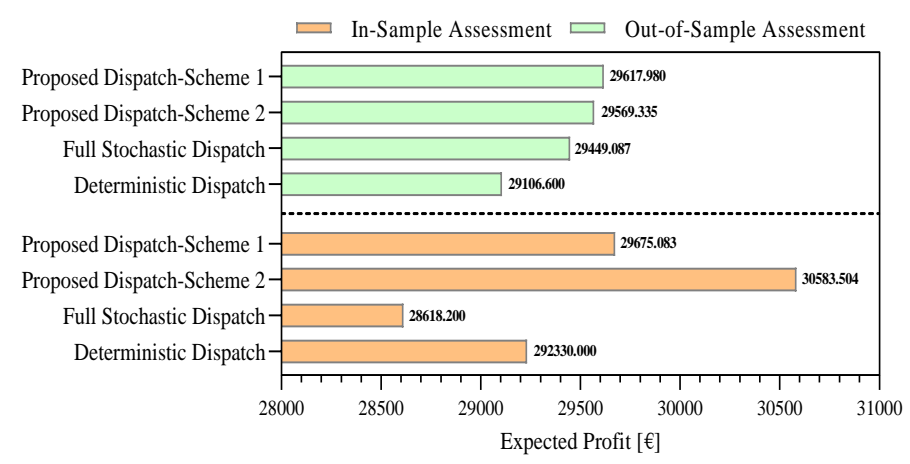

Fig. 12: In-sample and out-of-sample assessments for different approaches.

such integrated model. To develop the day-ahead and intraday dispatch problem under uncertainty, a comprehensive and correct CVaR-IGDT model was proposed, letting the decisionmakers to follow risk-seeker and risk-averse attitudes against the present uncertainties. The numerical results showed that: 1) The integrated dispatch of biomass and concentrated solar units brings substantial profitability compared to the individual dispatch of units; 2) The proposed CVaR-IGDT model serves as a comprehensive risk management model, allowing the decision-maker to pursue risk-averse actions against stochastic inputs while seeking either risk-seeker or risk-averse actions toward the IGDT input; 3) Despite the higher computation time of the proposed CVaR-IGDT model compared to conventional approaches, it is still a competitive and satisfactory model due to its better performance in the out-of-sample assessment; 4) As the out-of-sample test revealed, the proposed CVaR-IGDT model performs better than deterministic and full stochastic approaches.

As a future research direction, thermodynamic characteristics of the IBCS system will be modeled, and the resulting consequence on trading strategies will be explored.

\section{APPENDIX A}

The parameters of the day-ahead and intraday prices for the scenario generation process using normal distribution are presented in Table 6 and Table 7 . As stated in section VI the difference between day-ahead and intraday prices is leveraged to capture the correlation between stochastic inputs. This difference is indicated by defining a new parameter $\varkappa_{t}=$ $\sigma_{t}^{\mathrm{I}}-\sigma_{t}^{\mathrm{DA}}$. The mean values reported in Table 6 and Table 7 are referred to the Iberian electricity market on November 18,2019 , while standard deviations were obtained through the whole year analysis.

\section{APPENDIX B}

The algorithm of risk-controlling scheme 2 is mapped out in Algorithm 3

\section{APPENDIX C}

The characteristics of the IBCS system is reported in Table 4 The hourly forecasted thermal power of the solar field is illustrated in Table VIII.
TABLE V: Mean and Standard Deviation (STD) of Day-Ahead Price $[€ / M W h]$.

\begin{tabular}{|c|c|c|c|c|c|}
\hline$t$ & Mean $\sigma_{t}^{\text {DA }}$ & STD $\sigma_{t}^{\text {DA }}$ & $t$ & Mean $\sigma_{t}^{\text {DA }}$ & STD $\sigma_{t}^{\text {DA }}$ \\
\hline 1 & 40.74 & 11.16 & 13 & 50.77 & 9.75 \\
\hline 2 & 39.69 & 11.28 & 14 & 50.00 & 9.86 \\
\hline 3 & 36.89 & 11.52 & 15 & 50.45 & 10.10 \\
\hline 4 & 35.68 & 11.52 & 16 & 52.23 & 10.01 \\
\hline 5 & 39.11 & 11.38 & 17 & 54.54 & 10.06 \\
\hline 6 & 46.05 & 11.06 & 18 & 60.52 & 9.83 \\
\hline 7 & 51.73 & 11.04 & 19 & 63.05 & 9.54 \\
\hline 8 & 51.05 & 10.70 & 20 & 62.55 & 8.92 \\
\hline 9 & 52.19 & 9.97 & 21 & 59.15 & 8.73 \\
\hline 10 & 51.49 & 9.84 & 22 & 54.54 & 9.03 \\
\hline 11 & 50.45 & 9.76 & 23 & 53.79 & 9.65 \\
\hline 12 & 50.47 & 9.72 & 24 & 43.28 & 11.00 \\
\hline
\end{tabular}

TABLE VI: Mean and Standard Deviation (STD) of the Difference between Intraday and Day-Ahead Price [€/MWh].

\begin{tabular}{|c|c|c|c|c|c|}
\hline$t$ & Mean $\varkappa_{t}^{*}$ & STD $\varkappa_{t}$ & $t$ & Mean $\varkappa_{t}$ & STD $\varkappa_{t}$ \\
\hline 1 & 1.37 & 3.88 & 13 & 0.21 & 2.13 \\
\hline 2 & 1.2 & 3.82 & 14 & 0.67 & 2.40 \\
\hline 3 & 2.68 & 3.91 & 15 & 0.53 & 2.40 \\
\hline 4 & -1.61 & 3.32 & 16 & 0.40 & 2.34 \\
\hline 5 & 1.00 & 3.10 & 17 & 1.00 & 2.38 \\
\hline 6 & -4.86 & 2.86 & 18 & 3.74 & 2.29 \\
\hline 7 & -0.51 & 2.52 & 19 & 1.21 & 2.12 \\
\hline 8 & 1.51 & 2.28 & 20 & 0.43 & 2.16 \\
\hline 9 & 0.47 & 2.31 & 21 & -3.01 & 2.10 \\
\hline 10 & 0.81 & 2.36 & 22 & -0.50 & 2.20 \\
\hline 11 & 0.22 & 2.26 & 23 & -4.00 & 2.18 \\
\hline 12 & 0.51 & 2.10 & 24 & -0.77 & 5.09 \\
\hline & $* \varkappa_{t}=\sigma_{t}^{\text {I }}-\sigma_{t}^{\text {DA }}$ & &
\end{tabular}

TABLE VIII: Forecasted Thermal Power of Solar Field [MW].

\begin{tabular}{|c|c|c|c|c|c|c|c|}
\hline$t$ & $\bar{G}_{t}^{\mathrm{f}}$ & $t$ & $\bar{G}_{t}^{\mathrm{f}}$ & $t$ & $\bar{G}_{t}^{\mathrm{f}}$ & $t$ & $\bar{G}_{t}^{\mathrm{f}}$ \\
\hline 1 & 0.00 & 7 & 0.00 & 13 & 70.00 & 19 & 20.00 \\
\hline 2 & 0.00 & 8 & 20.00 & 14 & 80.00 & 20 & 0.00 \\
\hline 3 & 0.00 & 9 & 40.00 & 15 & 80.00 & 21 & 0.00 \\
\hline 4 & 0.00 & 10 & 80.00 & 16 & 80.00 & 22 & 0.00 \\
\hline 5 & 0.00 & 11 & 80.00 & 17 & 80.00 & 23 & 0.00 \\
\hline 6 & 0.00 & 12 & 50.00 & 18 & 50.00 & 24 & 0.00 \\
\hline
\end{tabular}

\section{REFERENCES}

[1] L. Cutz, G. Berndes, and F. Johnsson, "A techno-economic assessment of biomass co-firing in Czech Republic, France, Germany and Poland," Biofuels, Bioprod. Biorefining, vol. 13, no. 5, pp. 1289-1305, 2019.

[2] J. Li, J. Fang, Q. Zeng, and Z. Chen, "Optimal operation of the integrated electrical and heating systems to accommodate the intermittent renewable sources," Appl. Energy, vol. 167, pp. 244-254, 2016.

[3] E. Panos and R. Kannan, "The role of domestic biomass in electricity, heat and grid balancing markets in Switzerland," Energy, vol. 112, pp. $1120-1138,2016$.

[4] C. S. Lai and M. D. McCulloch, "Sizing of stand-alone solar PV and storage system with anaerobic digestion biogas power plants," IEEE Trans. Ind. Electron., vol. 64, no. 3, pp. 2112-2121, 2016.

[5] C. Li et al., "Optimal planning of islanded integrated energy system with solar-biogas energy supply," IEEE Trans. Sustain. Energy, vol. 11, no. 4 , pp. $2437-2448,2019$. 
Algorithm 3 Algorithm for Risk-Controlling Scheme 2.

1: Get the set of in-sample scenarios obtained from step 1 of Algorithm 1.

2: Optimize objective function (1) subject to constraints (2)(29) using in-sample scenarios to get the value of $\Pi_{0}$.

3: Set the values of confidence level $\kappa$ and expected profit deviation factor $\beta^{\text {scheme }_{2}}$.

4: Specify the objective functions involved in the optimization process. For risk-controlling scheme 2, the objectives are the minimization of $\alpha^{\text {scheme }_{2}}$ and maximization of $\mathrm{CVaR}_{\kappa}$ denoted by (47) and (31), respectively.

5: Acquire the range of objective function $\alpha^{\text {scheme }_{2}}$ leveraging the lexicographic principle. To do so, three following optimization problems must be solved.

$$
\begin{aligned}
& \Omega_{4}=\underset{\Psi, Q_{s}}{\operatorname{Maximize}}\left\{\mathrm{CVaR}_{\kappa}\right\} \\
& \text { s.t. [31, (32), (48) - (51), } Q_{s} \geq 0 \quad \forall s \\
& \left.\left.\Omega_{5}=\underset{\left[\xi_{t, s}^{\mathrm{DA}}, \xi_{t, s}^{\mathrm{I}}, m_{t, s}^{\mathrm{b}}\right] \cup \tilde{G}_{t}^{\mathrm{f}}}{\operatorname{Minimize}}\left\{\alpha^{\text {scheme }_{2}}\right\} \quad \text { s.t. } 48\right\}-51\right] \\
& \Omega_{6}=\underset{\xi_{t, s}^{\mathrm{DA}}, \xi_{t, s}^{\mathrm{I}}, m_{t, s}^{\mathrm{b}}}{\operatorname{Minimize}}\left\{\alpha^{\text {scheme }_{2}} \mid \mathrm{CVaR}_{\kappa}=\Omega_{4}\right\} \\
& \text { s.t. } 31 \text { - 32, 48 - 51, } Q_{s} \geq 0 \quad \forall s
\end{aligned}
$$

Then, the range of $\alpha^{\text {scheme }_{2}}$ is equal to $\Omega_{6}-\Omega_{5}$.

6: Generate different optimal Pareto strategies for objectives specified in step 4 leveraging $\epsilon$-constraint method. This is accomplished by means of optimizing the following problem.

$$
\begin{array}{ll}
\underset{\xi_{t, s}^{\mathrm{DA}}, \xi_{t, s}^{\mathrm{I}}, m_{t, s}^{\mathrm{b}}, \Psi, Q_{s}, \vartheta}{\text { Maximize }} & \mathrm{CVaR}_{\kappa}+\frac{\vartheta}{\Omega_{6}-\Omega_{5}} \\
\text { s.t. } & \alpha^{\mathrm{scheme}_{2}}+\vartheta=N_{\epsilon} \\
& N_{\epsilon}=\Omega_{6}+\left(\frac{\Omega_{5}-\Omega_{6}}{p}\right) \times \epsilon, \quad \epsilon=0,1, \ldots, p \\
& \text { 31, 32, }, 48,-151, Q_{s} \geq 0 \quad \forall s, \quad \vartheta \geq 0
\end{array}
$$

In (59), $\vartheta$ refers to an extra variable. By selecting the desired value for $p, p+1$ Pareto strategies will be obtained.

[6] M. Shahidehpour et al., "Multistage Expansion Planning of Integrated Biogas and Electric Power Delivery System Considering the Regional Availability of Biomass," IEEE Trans. Sustain. Energy, 2020.

[7] I. Blanco, A. N. Andersen, D. Guericke, and H. Madsen, "A novel bidding method for combined heat and power units in district heating systems," Energy Syst., pp. 1-20, 2019.

[8] Y. Wang, S. Lou, Y. Wu, M. Miao, and S. Wang, "Operation strategy of a hybrid solar and biomass power plant in the electricity markets," Electr. Power Syst. Res., vol. 167, pp. 183-191, 2019.

[9] E. Du et al., "Operation of a high renewable penetrated power system with CSP plants: a look-ahead stochastic unit commitment model,' IEEE Trans. Power Syst., vol. 34, no. 1, pp. 140-151, 2018.

[10] Z. Wu, M. Zhou, J. Wang, E. Du, N. Zhang, and G. Li, "Profit-Sharing Mechanism for Aggregation of Wind Farms and Concentrating Solar Power," IEEE Trans. Sustain. Energy, 2020.

[11] Y. Zhao, Z. Lin, F. Wen, Y. Ding, J. Hou, and L. Yang, "Risk-Constrained Day-Ahead Scheduling for Concentrating Solar Power Plants With Demand Response Using Info-Gap Theory," IEEE Trans. Ind. Informatics, vol. 15, no. 10, pp. 5475-5488, 2019.

[12] H. Khaloie, M. Mollahassani-pour, and A. Anvari-Moghaddam, "Optimal Behavior of a Hybrid Power Producer in Day-Ahead and Intraday
TABLE VII: Parameters of the IBCS System.

\begin{tabular}{|c|c|c|}
\hline Parameter & Value & Unit \\
\hline$C^{\mathrm{b}}$ & 54.65 & $€ /$ ton \\
\hline$C a^{\mathrm{b}}$ & 4.067 & $\mathrm{MWh} /$ ton \\
\hline$\overline{G G}$ & 150 & $\mathrm{MW}$ \\
\hline$M^{\mathrm{b}}$ & 500 & ton \\
\hline$t^{\text {off, bo }}, t^{\text {off }, \mathrm{pb}}$ & 2,2 & $\mathrm{hr}$ \\
\hline$t^{\mathrm{on}, \mathrm{bo}}, t^{\mathrm{on}, \mathrm{pb}}$ & 2,2 & $\mathrm{hr}$ \\
\hline$\gamma^{\mathrm{SU}}$ & 25 & $\mathrm{MW}$ \\
\hline$\gamma_{0}^{\mathrm{TS}}$ & $50 \% \overline{\Gamma^{\mathrm{Ts}}}$ & $\mathrm{MWh}$ \\
\hline$\Gamma^{\mathrm{bo}}, \overline{\Gamma^{\mathrm{bo}}}$ & 0,150 & $\mathrm{MW}$ \\
\hline$\underline{\Gamma^{\mathrm{pb}}}, \overline{\Gamma^{\mathrm{pb}}}$ & 50,250 & $\mathrm{MW}$ \\
\hline$\Gamma^{\mathrm{Ts}}, \overline{\Gamma^{\mathrm{Ts}}}$ & 120,1200 & $\mathrm{MWh}$ \\
\hline$\Delta^{\mathrm{down}}, \Delta^{\mathrm{up}}$ & $40 \% \overline{\Xi^{\mathrm{pb}}}, 40 \% \overline{\Xi^{\mathrm{pb}}}$ & $\mathrm{MW} / \mathrm{hr}$ \\
\hline$\overline{\zeta^{\mathrm{ch}}}, \overline{\zeta^{\mathrm{dis}}}$ & 150,150 & $\mathrm{MW}$ \\
\hline$\eta^{\mathrm{bo}}$ & 89.5 & $\%$ \\
\hline$\eta^{\mathrm{pb}}$ & 40 & $\%$ \\
\hline$\eta^{\mathrm{ch}}, \eta^{\mathrm{dis}}$ & 98 & $\%$ \\
\hline$\rho$ & 0.031 & $\%$ \\
\hline$\Upsilon$ & 0.3 & - \\
\hline
\end{tabular}

Markets: A Bi-Objective CVaR-Based Approach,” IEEE Trans. Sustain. Energy, p. 1, 2020.

[13] Y. Zhao, S. Liu, Z. Lin, F. Wen, L. Yang, and Q. Wang, "A Mixed CVaRBased Stochastic Information Gap Approach for Building Optimal Offering Strategies of a CSP Plant in Electricity Markets," IEEE Access, vol. 8, pp. 85772-85783, 2020.

[14] IRENA, Renewable Energy Technologies: Cost Analysis Series Biomass for Power Generation. 2012.

[15] R. Soria, J. Portugal-Pereira, A. Szklo, R. Milani, and R. Schaeffer, "Hybrid concentrated solar power (CSP)-biomass plants in a semiarid region: A strategy for CSP deployment in Brazil," Energy Policy, vol. 86, pp. 57-72, 2015.

[16] C. M. I. Hussain, B. Norton, and A. Duffy, "Comparison of hybridizing options for solar heat, biomass and heat storage for electricity generation in Spain," Energy Convers. Manag., vol. 222, p. 113231, 2020.

[17] R. Milani, A. Szklo, and B. S. Hoffmann, "Hybridization of concentrated solar power with biomass gasification in Brazil's semiarid region," Energy Convers. Manag., vol. 143, pp. 522-537, 2017.

[18] P. Morrone, A. Algieri, and T. Castiglione, "Hybridisation of biomass and concentrated solar power systems in transcritical organic Rankine cycles: A micro combined heat and power application," Energy Convers. Manag., 2019, doi: 10.1016/j.enconman.2018.11.029.

[19] A. J. Conejo, M. Carrión, and J. M. Morales, Decision Making Under Uncertainty in Electricity Markets, vol. 1. Boston, MA: Springer US, 2010.

[20] M. Ahmadigorji, N. Amjady, and S. Dehghan, "A Robust Model for Multiyear Distribution Network Reinforcement Planning Based on Information-Gap Decision Theory," IEEE Trans. Power Syst., 2018.

[21] H. Khaloie, A. Anvari-Moghaddam, N. Hatziargyriou, and J. Contreras, "Risk-constrained self-scheduling of a hybrid power plant considering interval-based intraday demand response exchange market prices," J. Clean. Prod., vol. 282, p. 125344, 2021.

[22] L. P. Garcés and A. J. Conejo, "Weekly self-scheduling, forward contracting, and offering strategy for a producer," IEEE Trans. Power Syst., vol. 25, no. 2, pp. 657-666, 2010.

[23] A. Arrigo, C. Ordoudis, J. Kazempour, Z. De Grève, J. F. Toubeau, and F. Vallée, "Optimal Power Flow under Uncertainty: An Extensive Outof-Sample Analysis," 2019, doi: 10.1109/ISGTEurope.2019.8905752.

[24] H. Khaloie et al., "Coordinated wind-thermal-energy storage offering strategy in energy and spinning reserve markets using a multi-stage model," Appl. Energy, vol. 259, p. 114168, Feb. 2020.

[25] G. Mavrotas, "Effective implementation of the $\epsilon$-constraint method in Multi-Objective Mathematical Programming problems," Appl. Math. Comput., vol. 213, no. 2, pp. 455-465, 2009. 


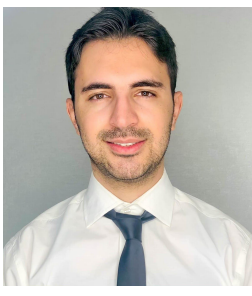

Hooman Khaloie received the M.Sc. degree (First Class Hons.) from Shahid Bahonar University of Kerman, Kerman, Iran, in 2019. During 2019 and 2021, he was a Research Assistant at Kerman Regional Electric Company, Iran's Ministry of Energy. He received the Outstanding Reviewer Award from the IEEE Transactions on Power Systems in 2020 His research interests include electricity markets, financial risk assessment, integrated energy systems, and operations research.

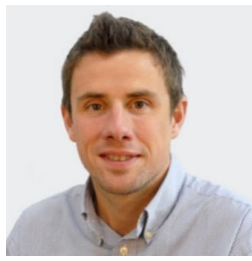

François Vallée (Member, IEEE) received the degree in civil electrical engineering and the Ph.D. degree in electrical engineering from the Faculty of Engineering, University of Mons, Belgium, in 2003 and 2009, respectively. He is currently a Professor and leader of the "Power Systems and Markets Research Group" at the University of Mons. His Ph.D. work has been awarded by the SRBE/KBVE Robert Sinave Award in 2010. His research interests include PV and wind generation modeling for electrical system reliability studies in presence of dispersed

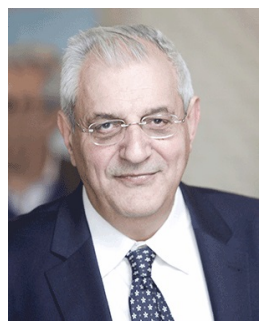

Nikos D. Hatziargyriou (Fellow, IEEE) is professor in Power Systems at the National Technical University of Athens. He has over 10 year industrial experience as Chairman and CEO of the Hellenic Distribution Network Operator (HEDNO) and as executive Vice-Chair and Deputy CEO of the Public Power Corporation (PPC), responsible for the Transmission and Distribution Divisions. He was chair and vice-chair of the EU Technology and Innovation Platform on Smart Networks for Energy Transition (ETIP-SNET) representing E.DSO. He is honorary member of CIGRE and past Chair of CIGRE SC C6 "Distribution Systems and Distributed Generation". He is Life Fellow Member of IEEE, past Chair of the Power System Dynamic Performance Committee (PSDPC) and currently Editor in Chief of the IEEE Trans on Power Systems. He is the 2017 recipient of the IEEE/PES Prabha S. Kundur Power System Dynamics and Control Award. He has participated in more than $60 \mathrm{RD} \& \mathrm{D}$ projects funded by the EU Commission, electric utilities and manufacturers for both fundamental research and practical applications. He is author of the book "Microgrids: Architectures and Control" and of more than 250 journal publications and 500 conference proceedings papers. He is included in the 2016, 2017 and 2019 Thomson Reuters lists of the top 1\% most cited researchers and he is 2020 Globe Energy Prize laureate.

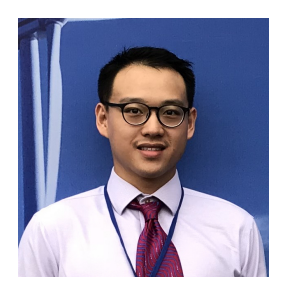

Chun Sing Lai (Senior Member, IEEE) received the B.Eng. (First Class Hons.) in electrical and electronic engineering from Brunel University London, London, U.K., in 2013, and the D.Phil. in engineering science from the University of Oxford, Oxford, U.K., in 2019. He is currently a Lecturer with the Department of Electronic and Electrical Engineering, Brunel University London. From 2018 to 2020, he was an Engineering and Physical Sciences Research Council Research Fellow with the School of Civil Engineering, University of Leeds, Leeds, U.K. His current research interests are in power system optimization and data analytics. Dr. Lai was the Secretary of the IEEE Smart Cities Publications Committee, the Acting Editor-in-Chief of IEEE Smart Cities Newsletters, and Publications Co-Chair for 2020 and 2021 IEEE International Smart Cities Conference. He is the Vice-Chair of the IEEE Smart Cities Publications Committee. He is the Working Group Chair for IEEE P2814 Standard, and the Chair of the IEEE SMC Intelligent Power and Energy Systems Technical Committee. He is an IET Member and a Chartered Engineer.

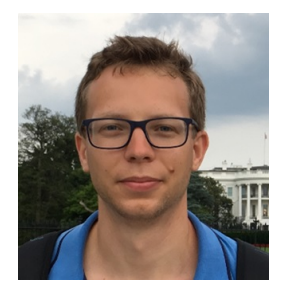

Jean-François Toubeau (Member, IEEE) received the degree in civil electrical engineering, and the $\mathrm{Ph} . \mathrm{D}$. degree in electrical engineering, from the University of Mons (Belgium) in 2013 and 2018, respectively. He is currently a postdoctoral researcher of the Belgian Fund for Research (F.R.S/FNRS) within the "Power Systems and Markets Research Group" of the same University. His research mainly focuses on bridging the gap between machine learning and decision-making in modern power systems. 